\title{
The circumstellar envelope of the C-rich post-AGB star HD 56126`
}

\author{
S. Hony ${ }^{1,2}$, A. G. G. M. Tielens ${ }^{3,4}$, L. B. F. M. Waters ${ }^{1,5}$, and A. de Koter ${ }^{1}$ \\ 1 Astronomical Institute "Anton Pannekoek", Kruislaan 403, 1098 SJ Amsterdam, The Netherlands \\ 2 RSSD-ESA/ESTEC, PO Box 299, 2200 AG Noordwijk, The Netherlands \\ 3 SRON Laboratory for Space Research Groningen, PO Box 800, 9700 AV Groningen, The Netherlands \\ ${ }^{4}$ Kapteyn Astronomical Institute, PO Box 800, 9700 AV Groningen, The Netherlands \\ 5 Instituut voor Sterrenkunde, KU Leuven, Celestijnenlaan 200B, 3001 Heverlee, Belgium
}

Received 12 August 2002 / Accepted 14 February 2003

\begin{abstract}
We present a detailed study of the circumstellar envelope of the post-asymptotic giant branch " $21 \mu \mathrm{m}$ object" HD 56126. We build a detailed dust radiative transfer model of the circumstellar envelope in order to derive the dust composition and mass, and the mass-loss history of the star. To model the emission of the dust we use amorphous carbon, hydrogenated amorphous carbon, magnesium sulfide and titanium carbide. We present a detailed parametrisation of the optical properties of hydrogenated amorphous carbon as a function of $\mathrm{H} / \mathrm{C}$ content. The mid-infrared imaging and spectroscopy is best reproduced by a single dust shell from 1.2 to $2.6^{\prime \prime}$ radius around the central star. This shell originates from a short period during which the mass-loss rate exceeded $10^{-4} M_{\odot} / \mathrm{yr}$. We find that the strength of the " 21 " $\mu \mathrm{m}$ feature poses a problem for the TiC identification. The low abundance of Ti requires very high absorption cross-sections in the ultraviolet and visible wavelength range to explain the strength of the feature. Other nano-crystalline metal carbides should be considered as well. We find that hydrogenated amorphous carbon in radiative equilibrium with the local radiation field does not reach a high enough temperature to explain the strength of the 3.3-3.4 and 6-9 $\mu \mathrm{m}$ hydrocarbon features relative to the 11-17 $\mu \mathrm{m}$ hydrocarbon features. We propose that the carriers of these hydrocarbon features are not in radiative equilibrium but are transiently heated to high temperature. We find that 2 per cent of the dust mass is required to explain the strength of the " 30 " $\mu \mathrm{m}$ feature, which fits well within the measured atmospheric abundance of $\mathrm{Mg}$ and $\mathrm{S}$. This further strengthens the $\mathrm{MgS}$ identification of the "30" $\mu \mathrm{m}$ feature.
\end{abstract}

Key words. stars: individual: HD 56126 - stars: AGB and post-AGB - stars: carbon - circumstellar matter stars: mass-loss - infrared: stars

\section{Introduction}

Post-asymptotic giant branch stars (post-AGBs) are objects in which the strong AGB mass-loss has ceased while the remaining star is not (yet) hot enough to ionise the surrounding material. These objects are characterised by a double-peaked spectral energy distribution (SED). In the ultraviolet (UV) and visible range the central star is visible. In the mid-infrared (IR) range the light is dominated by emission from the dusty circumstellar envelope (CSE). Post-AGB stars are the prime objects to study stellar evolution near the very end of the AGB because they are the most recent evolutionary descendants of the AGB stars. Mass loss near the tip of the AGB is not well understood. There are indications that the mass-loss rates near the end of the AGB exceed the values predicted by

Send offprint requests to: S. Hony, e-mail: shony@rssd.esa.int

* Based on observations taken at the European Southern Observatory, La Silla, Chile and observation obtained with ISO, an ESA project with instruments funded by ESA Member states (especially the PI countries: France, Germany, The Netherlands and the UK) with the participation of ISAS and NASA. the existing dust driven wind models (e.g. CRL 2688). There is also increasing evidence that non-spherical mass loss is common near the end of the AGB phase. Since AGB evolution timescales are determined by mass loss, these poorly understood phenomena limit our knowledge of the evolution of these objects and the subsequent stages.

Mass loss determinations are directly coupled to the composition of the newly condensed dust. The dust composition of the CSE reflects the physical conditions in the outer atmospheres of the AGB star during the phase when the dust was formed and the processing that took place afterwards. We study the composition of the dust surrounding the post-AGB star HD 56126. This star is member of a class of rare objects that exhibit an emission feature at $21 \mu \mathrm{m}$ (Kwok et al. 1989). The 12 (see Kwok et al. 1999, and references therein) post-AGB stars that show this feature are commonly referred to as the " $21 \mu \mathrm{m}$ objects". The " $21 \mu \mathrm{m}$ objects" form a group with rather homogeneous properties: carbon-rich, low metallicity and s-process enhanced (van Winckel \& Reyniers 2000) with large IR excesses (Kwok et al. 1989), spectral types G or F (Hrivnak 1995). Volk et al. (1999) have shown that the shape 
of the " 21 " $\mu \mathrm{m}$ feature between different sources shows hardly any variation. Recently we have reported the detection of the same feature in two PNe (Hony et al. 2001) and Volk et al. (2002) have reported on its detection in the PN IC 418.

Besides the " 21 " $\mu \mathrm{m}$ feature the " $21 \mu \mathrm{m}$ objects" exhibit other infrared emission features that are unique to these environments. The carriers of many of these features have not been firmly identified and there is considerable debate in the literature on the composition of the dust around the " $21 \mu \mathrm{m}$ objects". In the next paragraphs we shortly review these features and the proposed carriers.

The carrier of the " 21 " $\mu \mathrm{m}$ feature has remained unidentified for more than a decade, although several candidates have been considered: urea OC $\left(\mathrm{NH}_{2}\right)_{2}$ (Sourisseau et al. 1992), $\mathrm{SiS}_{2}$ (Goebel 1993; Begemann et al. 1996), fulleranes (Webster 1995), nano-diamond (Hill et al. 1998), hydrogenated amorphous carbon (HAC) (Grishko et al. 2001) and oxygen bearing side groups in coal (Papoular 2000). However, none of the spectroscopic comparisons between the laboratory spectra of the suggested materials and the emission from the postAGB stars is satisfactory. Recently we have suggested titanium carbide nano-crystals (nano-TiC) as the carrier of the " 21 " $\mu \mathrm{m}$ feature (von Helden et al. 2000). This identification is based on i) the excellent spectroscopic match of nano-TiC with the $21 \mu \mathrm{m}$ feature, ii) the fact that the nano- $\mathrm{TiC}$ resonance does not depend on the crystal size for the size-range for which measurements are available - in good agreement with its observed constant profile, iii) the fact that $\mathrm{TiC}$ is found in presolar grains (Bernatowicz et al. 1996) for which the isotopic ratios imply an origin in the CSE of carbon-rich evolved stars (Anders \& Zinner 1993).

We note that the nano-TiC identification is not commonly accepted either. The main counter argument is that the conditions (high density and pressure during dust formation) required to form $\mathrm{TiC}$ in the ejecta of carbon-rich evolved stars may not be met (Kwok et al. 2002). Another argument against nano-TiC is the strength of the " 21 " $\mu \mathrm{m}$ feature. Since $\mathrm{Ti}$ is a rare element, it may be difficult for $\mathrm{TiC}$ to produce a feature that stands out as much as it does.

As explained above the " $21 \mu \mathrm{m}$ objects" exhibit other emission features for which the carriers are still under debate. Besides the well-known unidentified infrared (UIR) bands at $3.3,6.2, " 7.7 "$ and 11.2, generally attributed to polycyclic aromatic hydrocarbon (PAH) molecules (Allamandola et al. 1989), these sources exhibit a $3.4 \mu \mathrm{m}$ feature and broad emission features (so-called plateau features) between 6-9 and 11-17 $\mu \mathrm{m}$ (Buss et al. 1990; Justtanont et al. 1996). As a class, the emission bands observed from the " $21 \mu \mathrm{m}$ objects" are distinctly different from the UIR features from most sources (see Sect. 3.1). Kwok et al. (2001) have discussed these features in terms of alkane and alkene side groups on very large aromatic molecules and small carbonaceous particles. Guillois et al. (1996) have compared them to those measured in coal (a natural terrestrial carbonaceous compound). The spectroscopic match with coal is very encouraging. However, a fundamental questions concerning the identification is whether carbonaceous grains can reach the temperatures required to emit strongly in the $6-9 \mu \mathrm{m}$ range.
Lastly, these sources exhibit a strong, broad feature between 23-45 $\mu \mathrm{m}$ (Omont et al. 1995; Szczerba et al. 1997; Hrivnak et al. 2000) called the " 30 " $\mu \mathrm{m}$ feature. The carrier of the "30" $\mu \mathrm{m}$ feature is identified with magnesium sulfide (MgS) (See Hony et al. 2002, and references therein). The identification of the " 30 " $\mu \mathrm{m}$ feature with $\mathrm{MgS}$ seems solid but open questions remain on the heating of the $\mathrm{MgS}$ grains and the amount of $\mathrm{MgS}$ required to explain the prominence of the feature.

A meaningful approach towards the identification of the various dust components in the CSE of these post-AGBs is that of radiative transfer modelling of the SED of these objects. This may also help to improve our understanding of the mass loss at the very end of the AGB phase. Such modelling allows a comparison of the emission characteristics of candidate materials and CSE emission and yields insight into the relative contributions of the different materials present in the CSE.

We have elected to model HD 56126 (IRAS 07134+1005, SAO 96709, BD+10 1470) in detail. HD 56126 has the strongest " 21 " $\mu \mathrm{m}$ feature relative to the other dust features of all known " $21 \mu \mathrm{m}$ objects". Therefore, HD 56126 provides the strongest constraint on the abundances needed to explain the " 21 " $\mu \mathrm{m}$ feature. This is even more so because the photosphere of the star is metal poor (van Winckel \& Reyniers 2000). Both the central star and the dust shell of this source have been well studied in the literature. Especially important in this respect are images at various wavelengths in the mid-IR in which the source is well resolved (Meixner et al. 1997; Dayal et al. 1998; Jura et al. 2000; Kwok et al. 2002, and this work). These images are essential to constrain the model parameters.

This paper is organised as follows: In Sect. 2 we give the stellar parameters of HD 56126. In Sect. 3 we present the infrared measurements that we use. In Sect. 4 we consider the energy balance in the nebula and constrain the stellar effective temperature. In Sect. 5 we list the parameters that we use to model the absorption and emission in the CSE of HD 56126. The optical properties of the dust constituents are discussed in Sect. 6. In Sect. 7 we present the model results. In Sect. 8 we discuss our findings and finally in Sect. 9 we give a summary and conclusions.

\section{Stellar parameters}

HD 56126 is a bright star with a visual magnitude of 8.3. The star has been classified as a F5 supergiant by Nassau et al. (1965). More recently Hrivnak et al. (1989) published a medium resolution optical spectrum and determined the spectral type to be F0-5I, consistent with the previous spectral classification. There are several photospheric abundance determinations published for HD 56126 (Parthasarathy et al. 1992; Klochkova 1995; van Winckel \& Reyniers 2000). These studies all demonstrate the low photospheric metallicity of the star, with a value of $[\mathrm{Fe} / \mathrm{H}]=-1.0$ and enrichment of $\mathrm{C}$, $\mathrm{N}, \mathrm{O}$ and the s-process elements. There is less agreement on the exact amount of enrichment of the CNO-elements. While Parthasarathy et al. (1992) and van Winckel \& Reyniers (2000) find a C/O value close to unity, Klochkova (1995) determine a lower abundance of $\mathrm{O}$ and derive a $\mathrm{C} / \mathrm{O}$ value of $\sim 1.5$. 
Table 1. UV to IR photometric data of HD 56126. Units are magnitudes unless given explicitly.

\begin{tabular}{|c|c|c|c|c|c|}
\hline \multicolumn{5}{|c|}{ Walraven } & \multirow{4}{*}{ 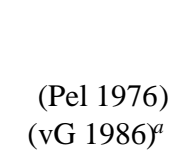 } \\
\hline V & $V-B$ & $B-U$ & $U-W$ & $B-L$ & \\
\hline-0.548 & 0.365 & 0.75 & 0.556 & 0.312 & \\
\hline \multirow[t]{2}{*}{-0.555} & 0.400 & 0.766 & 0.588 & 0.322 & \\
\hline & \multicolumn{5}{|c|}{ Johnson (van Genderen et al. 1986) } \\
\hline$B$ & V & & & & \\
\hline \multirow[t]{2}{*}{9.13} & 8.23 & & & & \\
\hline & \multicolumn{4}{|c|}{ Near-IR (Hrivnak et al. 1989) } & \\
\hline$J$ & $H$ & $K$ & $L$ & $M$ & \\
\hline \multirow[t]{2}{*}{6.92} & 6.66 & 6.65 & 6.43 & 6.10 & \\
\hline & \multicolumn{4}{|c|}{ Strömgren } & \\
\hline$b-y$ & \multirow{2}{*}{\multicolumn{2}{|c|}{$\begin{array}{l}(v-b)-(b-y) \\
0.182\end{array}$}} & \multirow{2}{*}{\multicolumn{2}{|c|}{$\begin{array}{l}(u-v)-(v-b) \\
1.477\end{array}$}} & \\
\hline 0.639 & & & & & (Olsen 1993) \\
\hline$u$ & $y$ & \multicolumn{3}{|c|}{$\left[\operatorname{ergs~s}^{-1} \mathrm{~cm}^{-2} \AA^{-1}\right]$} & \\
\hline \multirow[t]{2}{*}{$1.03 \mathrm{e}-12$} & $1.71 \mathrm{e}-12$ & & & \multicolumn{2}{|c|}{ (Ueta et al. 2000) } \\
\hline & \multicolumn{4}{|c|}{ Geneva (van Winckel, priv.comm.) } & \\
\hline$U$ & B & $B 1$ & $B 2$ & $V 1$ & $G$ \\
\hline 11.010 & 8.337 & 9.452 & 9.616 & 8.970 & 9.213 \\
\hline
\end{tabular}

${ }^{a}$ van Genderen et al. (1986).

The conversion from spectral type to effective temperature $\left(T_{\text {eff }}\right)$ is not clear-cut. Due to the peculiar abundances one should be critical of applying standard spectral type calibrations based on solar type abundances. For example, the tables of de Jager \& Nieuwenhuijzen (1987) quote 6370 for a F5I star with solar abundances. Values of $T_{\text {eff }}$ for HD 56126 within a large range (5900-7250 K) are derived by various methods. We give most weight to the temperatures determined in detailed abundance analyses using high resolution spectroscopy (Parthasarathy et al. 1992; van Winckel \& Reyniers 2000). These studies yield a high value of the effective temperature (7000-7250 K). This temperature corresponds to a F1-2I spectral classification, still within the range derived by Hrivnak et al. (1989). Such a high value for $T_{\text {eff }}$ also fits better with the observed spectral energy distribution (SED) (see below).

Jura et al. (2000) have made the case that the low iron abundance of the star, the high galactic latitude $\left(b=+10^{\circ}\right)$ and its high radial velocity make it probable that HD 56126 is a population II star with a zero age main-sequence mass of $\sim 1.1 \mathrm{M}_{\odot}$.

The distance $(d)$ to HD 56126 is poorly known. Values between 2 and $3 \mathrm{kpc}$ are used in the literature. Yuasa et al. (1999) derive a statistical distance of $2.12 \mathrm{kpc}$ from a principle component analysis based on IRAS photometry and the molecular expansion velocity. Knapp et al. (2000) derive $2.4 \mathrm{kpc}$ from the radial velocity assuming that the radial velocity is due to the galactic rotation while Jura et al. (2000) derive $2.3 \mathrm{kpc}$ from the theoretical luminosity of $6600 L_{\odot}$ and the observed flux. In the following we elected to use $d=2.4 \mathrm{kpc}$. Note that this distance yields a luminosity of $6000 L_{\odot}$ which is higher than expected ( 3000-4000 $L_{\odot}$ ) (e.g. Boothroyd \& Sackmann 1988) for a star with a $\sim 1.1 M_{\odot}$ ZAMS mass on the basis of the initial mass-final mass relation and core-mass-luminosity relation.
Table 2. Details of the ISO observations used in this study.

\begin{tabular}{llccc}
\hline \hline \multirow{2}{*}{ Instrument } & \multicolumn{3}{c}{ HD 56126 ISO observations } & \\
& $\begin{array}{l}\text { Obs. } \\
\text { mode }\end{array}$ & $\begin{array}{c}\alpha \\
(J 2000)\end{array}$ & $\begin{array}{c}\delta \\
(J 2000)\end{array}$ & TDT $^{b}$ \\
\hline SWS & 06 & 071610.20 & +095948.01 & 71802201 \\
SWS & 06 & 071610.30 & +095948.01 & 72201702 \\
SWS & $01(3)$ & 071610.20 & +095948.01 & 72201901 \\
LWS & 01 & 071610.20 & +095947.80 & 72201802 \\
\hline
\end{tabular}

a Observing mode used, see de Graauw et al. (1996) and Clegg et al. (1996). Numbers in brackets correspond to the scanning speed.

${ }^{b}$ TDT number which uniquely identifies each ISO observation.

In view of the uncertain distance, we explicitly give the distance dependence of the values that we derive.

\section{Observations and data reduction}

\subsection{Infrared spectroscopy}

For the spectral energy distribution (SED) of HD 56126 we use IR data obtained with the Short Wavelength Spectrometer (SWS) (de Graauw et al. 1996) and Long Wavelength Spectrometer (LWS) (Clegg et al. 1996) on-board the Infrared Space Observatory (ISO) (Kessler et al. 1996). These data consist of one SWS/AOT01 spectrum from 2.3-45 $\mu \mathrm{m}$, two SWS/AOT06 spectra covering the ranges 16.5-24 and 23$43 \mu \mathrm{m}$ and a LWS spectrum from 45 to $200 \mu \mathrm{m}$. Details of the ISO observations are given in Table 2.

The SWS data reduction steps involved the removal of cosmic ray hits. Next, we combine all available data from the SWS observations in order to maximise the signal-to-noise ratio. We correct each separate observation, subband and detector combination in such a way that its average equals the average of all available data at the corresponding wavelength. Below $12 \mu \mathrm{m}$ we apply an offset and above $12 \mu \mathrm{m}$ a scaling to the data. We apply an additional scaling factor of 1.1 to the data of subband $3 \mathrm{C}(16-19.5 \mu \mathrm{m})$ to form a continuous spectrum. Finally, the data are rebinned to a fixed resolution grid of $\lambda / \Delta \lambda=200$.

Data reduction of the LWS observation consisted of extensive bad data removal, rebinning on a fixed resolution grid of $\lambda / \Delta \lambda=50$ and splicing of the data to form a continuous spectrum. We apply scaling factors to the data from 45-70 $\mu \mathrm{m}$ and offsets at longer wavelengths. The data of detector $7(130-140 \mu \mathrm{m})$ are very bad and we have replaced those by a constant level of $10 \mathrm{Jy}$, the average of the much better data on either side of detector 7. The combined SWS/LWS spectrum together with data from the literature is presented in Fig. 1.

The SED of HD 56126 is double peaked. At wavelengths shorter than $4 \mu \mathrm{m}$ we see the stellar photosphere directly. At longer wavelengths the dust emission dominates. We see continuum emission which peaks at $\sim 25 \mu \mathrm{m}$ with strong broad emission features perched on top. In Fig. 1 we indicated the broad continuum due to amorphous carbon (a-carbon) and the " $21 " \mu \mathrm{m}$, " $30 " ~ \mu \mathrm{m}$ and the plateau feature, (tentatively) identified with titanium carbide, magnesium sulfide, and HAC or PAHs, respectively. 


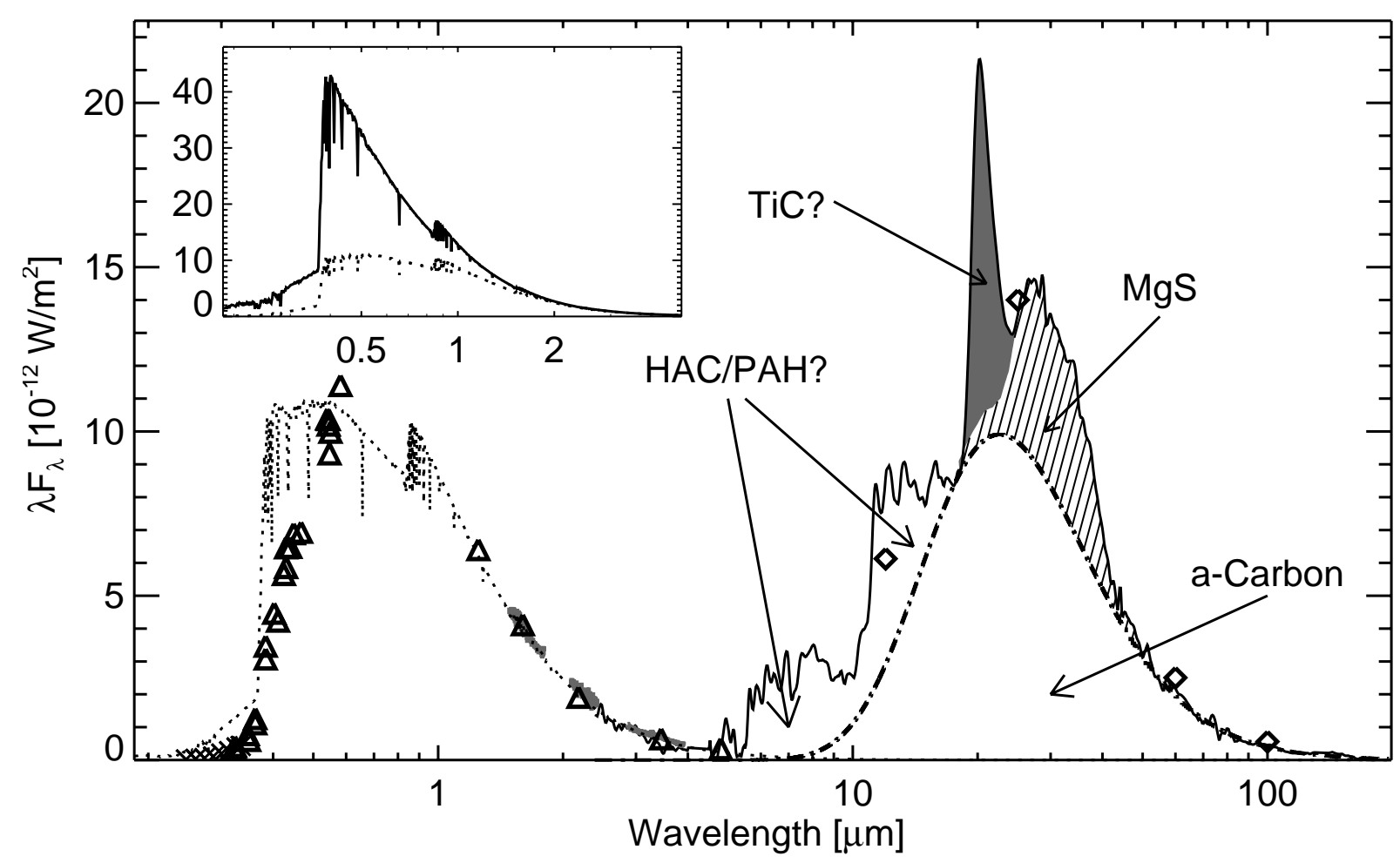

Fig. 1. The spectral energy distribution of HD 56126. We show the IUE data (crosses), optical and near-IR photometry (triangles) (see Table 1 for details), IRAS photometry (diamonds) and the ISO/SWS and ISO/LWS spectra (solid line). In grey we show near-IR spectroscopy from Kwok et al. (1990) and Oudmaijer et al. (1995). For reference we also show a Kurucz model (dotted line) of the central star with $T_{\text {eff }}=7250 \mathrm{~K}$, $\log g=1.0$ and $[\mathrm{M} / \mathrm{H}]=-1.0$ (Kurucz 1993) reddened with $E(B-V)=0.33$ using a standard extinction law (Savage \& Mathis 1979). The standard extinction law is clearly not applicable. In grey we indicate the contribution of the " 21 " $\mu \mathrm{m}$ feature and the dashed area indicates the " 30 " $\mu \mathrm{m}$ feature due to magnesium sulfide. Note that on this scale equal surface corresponds to equal energy. Therefore one may readily see that an appreciable amount of energy is emitted in the " 21 " and " 30 " $\mu \mathrm{m}$ features. The inset shows the Kurucz model before reddening (solid line) and the reddened model atmosphere (dotted line).

In Fig. 2 we show a detailed view of the plateau features. We indicate the emission that can be attributed to aromatic and aliphatic bonds separately. Notice the differences with the emission spectrum of the planetary nebula NGC 7027 in feature shape and relative strength of the bands. The aliphatic component is much stronger in HD 56126 than in NGC 7027.

\section{2. $N$-band imaging}

We also obtained an $11.9 \mu \mathrm{m}$ image with the TIMMI2 (Reimann et al. 2000; Käufl et al. 2000) mid-IR camera attached to the ESO $3.6 \mathrm{~m}$ telescope at La Silla, Chile on 25 December 2001. The camera is equipped with a $320 \times$ 240 pixel array; we applied a pixel scale of $0.3^{\prime \prime} /$ pixel. We used a chop throw of $15^{\prime \prime}$ north-south and a nod of $15^{\prime \prime}$ eastwest. This allowed for both the chopped as well as the nodded positions to fall onto the detector. We combined the positive and negative images resulting from the chopping and nodding positions. The images were reduced using a shift-and-add technique, where the shift between individual frames was determined from a least-squares comparison between the individual frames. The resulting image was then deconvolved using an empirically determined point spread function obtained by observing HD 32887 with the same set-up as HD 56126. We show the final image in Fig. 3.
The TIMMI2 $11.9 \mu \mathrm{m}$ image shows a clearly resolved envelope of $\sim 5^{\prime \prime}$ diameter. The emission peaks at $1.1^{\prime \prime}$ to the east from the centre of the nebulae. There are two other local intensity maxima: in the west and in the north, although the latter is not clearly resolved. Our $11.9 \mu \mathrm{m}$ image is consistent with other mid-IR imaging studies (Meixner et al. 1997; Dayal et al. 1998; Jura et al. 2000; Kwok et al. 2002) that show the same morphology throughout the $8-21 \mu \mathrm{m}$ wavelength range. The emission maximum in the north is resolved in the images of (2002). We derive the azimuthal averaged intensity profile from Fig. 3 to compare with the model output.

\section{Basic considerations}

Before we describe the details of our model we consider a few basic properties and the simple constraints that we can derive from them.

\subsection{Energy balance}

In Fig. 1 equal areas under the curve correspond to equal energy and it is clear that most of the energy is emitted in the IR. In Table 3 we specify the fractions of the energy emitted by each of the components in Fig. 1. Only 44 per cent of the light comes from the star directly while 56 per cent has been absorbed in the 


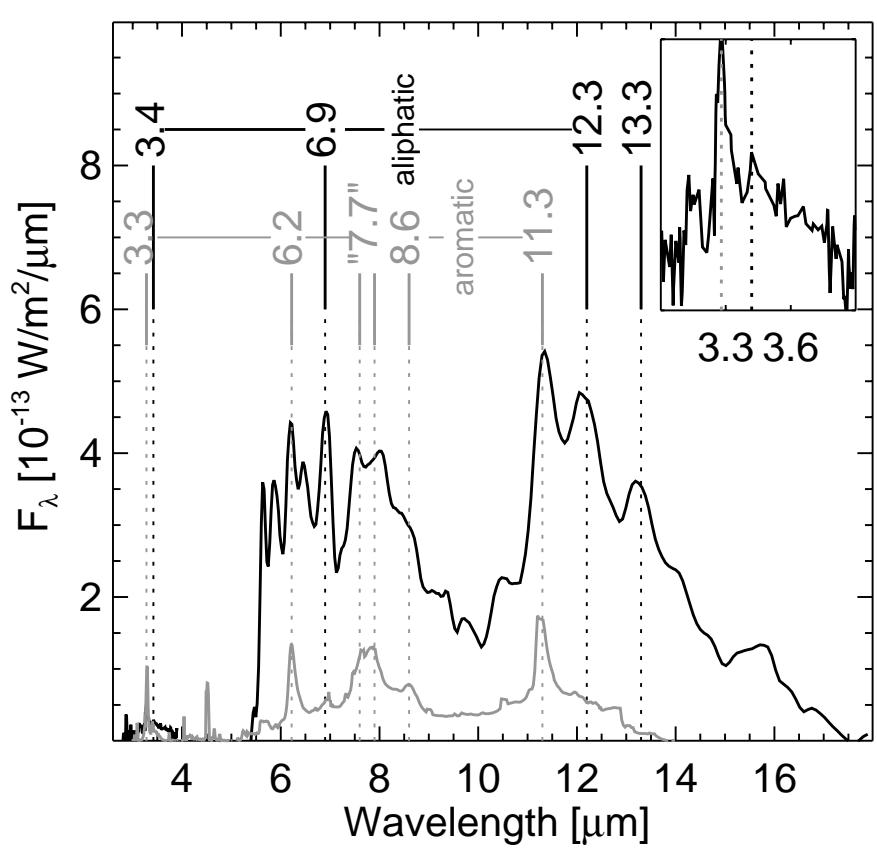

Fig. 2. The 3 to $17 \mu \mathrm{m}$ spectrum of HD 56126 after removing the contribution of the star and the dust continuum. Several emission features due to vibrationally excited $\mathrm{C}-\mathrm{H}$ and $\mathrm{C}-\mathrm{C}$ bonds in hydrogencarbon compounds are identified. We have indicated the features due to aromatic (grey lines) and aliphatic (black lines) structure. The inset shows a blow-up of the $3 \mu \mathrm{m}$ region, where the dominant aromatic $3.3 \mu \mathrm{m}$ and the weaker $3.42 \mu \mathrm{m}$ signature are observed. For reference we show the spectrum of the PN NGC 7027 as a prototypical PAH spectrum (grey). The emission features of HD 56126 are markedly different from the PAH spectrum of NGC 7027.

CSE and re-emitted in the IR. This implies that the extinction averaged over all angles around the star must be appreciable. Using a standard extinction law and a $7250 \mathrm{~K}$ Kurucz model atmosphere (Kurucz 1993) we estimate that the average $A_{\mathrm{V}}$ equals 1.1 , which corresponds to $\tau_{\mathrm{V}}=1.2$. However, such a value for the visual extinction corresponds to hardly any extinction in the near and mid-IR. Thus the near-IR $(2-4 \mu \mathrm{m})$ observations give the true continuum level of the star since no obvious dust contribution is detected in this wavelength range. Therefore, for a given effective temperature of the stellar photosphere there is no freedom in choosing the absolute scale. With this we can directly compare the integrated flux in the observed SED to the integrated flux from the model atmosphere. In Fig. 4 we show the integrated flux in the complete observed SED $\left(\mathcal{F}_{\text {obs }}\right)$ divided by the integrated flux in a Kurucz model atmosphere $\left(\mathcal{F}_{\text {atm }}\right)$ as a function of $T_{\text {eff }}$. As parameters of the Kurucz models we choose $\log g=1.0$ and a metallicity of $[\mathrm{Fe} / \mathrm{H}]=-1.0$ as determined for this star. For $T_{\text {eff }}$ in the range $7000-7500 \mathrm{~K} \mathcal{F}_{\text {obs }} / \mathcal{F}_{\text {atm }}$ is approximately one. This ratio should be close to unity if the following assumptions hold. i) The extinction in the near-IR is negligible. ii) The extinction along our line of sight is similar to the average value of the extinction.

The first assumption holds unless there is a large amount of grey extinction. Extinction caused by particles smaller than the wavelength at which they absorb falls off steeply when going to longer wavelengths. This is illustrated by the inset in Fig. 1

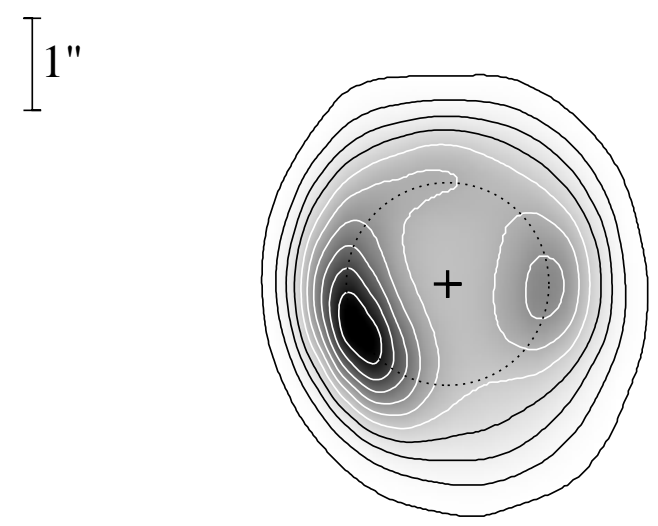

\section{$11.9 \mu \mathrm{m}$}

$\mathrm{E} \longleftarrow \stackrel{\mathrm{N}}{\uparrow}$

Fig. 3. TIMMI2 image of HD 56126 in the $11.9 \mu \mathrm{m}$ narrow band filter. The scale on the top left indicates $1^{\prime \prime}$. There are three emission maxima on a ring-like structure (indicated by a dotted line) at $\sim 1.1^{\prime \prime}$ from the centre (indicated by the "+"-sign). The grey-scale shows the square of the measured intensity to enhance the contrast of the emission maxima. The contours indicate the 5-95 per cent intensity levels in steps of 10 per cent. The outer contour ( 5 per cent) lies at $\sim 2.4^{\prime \prime}$ from the centre.

where we show a Kurucz model atmosphere with $T_{\text {eff }}=7250 \mathrm{~K}$ before and after reddening with standard interstellar extinction. The extinction in the UV and visible is substantial but the flux level at wavelengths beyond $2 \mu \mathrm{m}$ is hardly affected. Grey extinction is due to dust grains much larger than the wavelength at which they absorb. Typically these grains should have a diameter of at least several $\mu \mathrm{m}$ in order to cause wavelength independent extinction up to $4 \mu \mathrm{m}$. It is unlikely that a large amount of such big grains grow in stellar outflows. Moreover, the presence of these large dust grains would have strong implications for the understanding of the complete nebula, most notably the nebular mass budget. These implications are discussed in Sect. 8.3.

The second condition, concerning the extinction along the line of sight compared to the average extinction, is more debatable. The mid-IR images as published by Meixner et al. (1997), Dayal et al. (1998) and Jura et al. (2000) show clear deviations from spherical symmetry. These asymmetries are due to a nonspherical distribution of the dust around the star. We can write a simple expression for the flux observed from the system.

$$
\begin{aligned}
& \mathcal{F}_{\text {obs }}=\underbrace{\left(1-\eta_{\text {los }}\right) \mathcal{F}_{\text {atm }}}_{\text {UV +visible }}+\underbrace{\eta_{\text {aver }} \mathcal{F}_{\text {atm }}}_{\mathrm{IR}} \Leftrightarrow \\
& \frac{\mathcal{F}_{\text {obs }}}{\mathcal{F}_{\text {atm }}}=1-\eta_{\text {los }}+\eta_{\text {aver }}
\end{aligned}
$$

where $\eta_{\text {los }}$ is the fraction of the stellar flux absorbed in the line of sight towards the earth and $\eta_{\text {aver }}$ is the fraction of the stellar flux absorbed, averaged over all lines of sight from the star. In this we assume that the energy absorbed in the CSE is re-emitted isotropically in the IR. The energy budget equation is further constrained by the observational fact that $\eta_{\text {aver }}=1.25\left(1-\eta_{\text {los }}\right)$. This yields with Eq. (1) $\mathcal{F}_{\text {obs }} / \mathcal{F}_{\text {atm }}=$ $2.25-2.25 \eta_{\text {los }}$ 


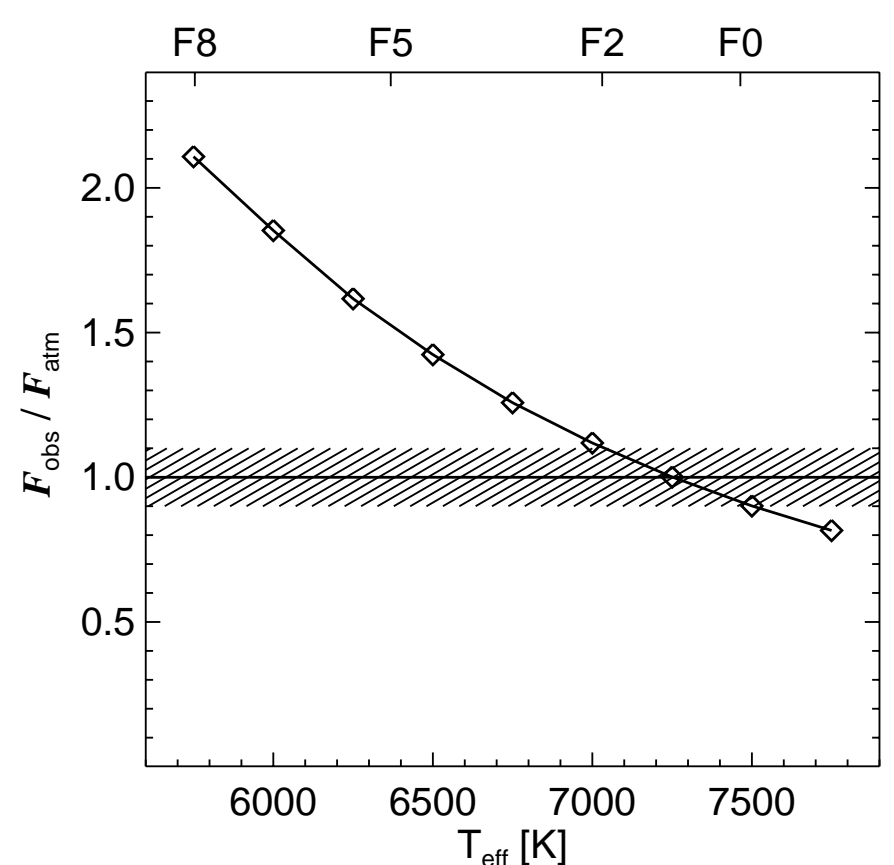

Fig. 4. The ratio of the observed integrated flux over the integrated flux from the Kurucz model atmosphere scaled to the near-IR observations versus the effective temperature of the model atmosphere. The hatched area indicates the range in which this ratio equals unity within the uncertainties. We indicate the position of the corresponding spectral types taken from de Jager \& Nieuwenhuijzen (1987) for supergiants along the top abscissa.

Table 3. The integrated contributions of the different components of the spectral energy distribution (see Fig. 1). We list the integrated flux (Col. 2), the fraction of the total luminosity (Col. 3) and the fraction of the IR luminosity (Col. 4).

\begin{tabular}{lccc}
\hline \hline Component & $\begin{array}{c}\text { Flux } \\
{\left[10^{-12} \mathrm{~W} / \mathrm{m}^{2}\right]}\end{array}$ & $\begin{array}{c}\text { Frac }_{\text {total }} \\
\%\end{array}$ & $\begin{array}{c}\text { Frac }_{\text {dust }} \\
\%\end{array}$ \\
\hline Total & 33.6 & 100 & - \\
Star & 14.8 & 44 & - \\
Dust & 18.8 & 56 & 100 \\
\hline \multicolumn{4}{c}{ Dust components } \\
\hline a-carbon & 11.6 & 35 & 62 \\
HAC/PAHs & 3.0 & 9 & 16 \\
MgS & 2.7 & 8 & 14 \\
TiC & 1.5 & 4 & 8 \\
\hline
\end{tabular}

In case $\eta_{\text {los }}<\eta_{\text {aver }}$ (i.e. when we are viewing the star relatively unobscured) $\mathcal{F}_{\text {obs }} / \mathcal{F}_{\text {atm }}$ is larger than unity. This is due to light that the star emits in directions away from the direction of the earth. When this light is absorbed in the CSE and partly re-emitted towards earth we measure extra flux. This would allow for a lower $T_{\text {eff }}$ than the $7000 \mathrm{~K}$ we derive from Fig. 4. However the observations put strong constraints on the importance of this effect. Mathematically $\eta_{\text {los }}$ and $\eta_{\text {aver }}$ can of course be in the range $0-1$, but in practise the UV and visible photometric data reveal a reddened star and $\eta_{\text {los }}$ must be larger than $\tau_{\mathrm{V}}(\sim 0.33)$. This is further corroborated by the fact that 56 per cent of the flux is emitted in the IR part of the spectrum. In Eq. (1) a value of $\eta_{\text {los }}$ lower than 0.33 requires $\eta_{\text {aver }}$ to be over 0.85 . This corresponds to the unlikely situation in which the star is completely surrounded by an opaque CSE with a small hole through which we happen to view the star unobscured. Reasonable values of $\eta_{\text {los }}$ are within $0.44-0.6$ which corresponds to $\mathcal{F}_{\text {obs }} / \mathcal{F}_{\text {atm }}=1.26-0.9$ and $T_{\text {eff }}=6800-7500 \mathrm{~K}$.

It is important to note that both Meixner et al. (1997) and Dayal et al. (1998) have constructed models for HD 56126 in which $\eta_{\text {los }}>\eta_{\text {aver }}$ in order to reproduce the observed midIR morphology. This yields a $T_{\text {eff }}$ in the high end tail of the range mentioned above. Likewise, interstellar extinction can be considered as an added contribution to $\eta_{\text {los }}$ also yielding a higher effective temperature for the central star. Bogaert (1994, chapter 3) has observed stars in the vicinity of HD 56126 and estimates the interstellar contribution to the reddening to be $E(B-V)_{\mathrm{IS}}=0.04$. We conclude that a high effective temperature in the range $7000-7500 \mathrm{~K}$ fits best with all observed properties of HD 56126. In the following we use $T_{\text {eff }}=7250 \mathrm{~K}$ as derived by van Winckel \& Reyniers (2000).

\subsection{Feature strength}

What is also directly apparent from Fig. 1 is the large fraction of the light emitted in the IR features. We have indicated the HAC/PAH features and the " 21 " and " 30 " $\mu \mathrm{m}$ features. Together these features carry 40 per cent of the total IR flux (see also Table 3). Any self-consistent radiative transfer modelling of the nebula must take absorption and emission by the feature carriers into account. The reason for this is twofold. First, the feature carriers cause an important part of the reddening of the star. Thus, when comparing model results to the observed photometry these are essential to assess the validity of the model. Second, in the CSE these components absorb a sizeable part of the stellar light and thus determine, in part, the temperature structure of the CSE as a whole. The temperature structure of the CSE is a very important parameter that translates directly into observables like the mid-IR spectrum and mid-IR surface brightness.

The strength of the IR features as seen in Fig. 1 also exposes the enigma of this nebula. Several of the observed features are supposedly carried by materials composed of elements with low abundances. The key question is: "Is it possible that trace species can cause such strong features?". Note that this question applies not only to TiC concerning the " 21 " $\mu \mathrm{m}$ feature but similarly to the " 30 " $\mu \mathrm{m}$ feature which is produced by $\mathrm{MgS}$. While $\mathrm{Mg}$ and $\mathrm{S}$ are both more abundant than Ti they are relatively unimportant in comparison to C (van Winckel \& Reyniers 2000) that causes the continuum.

\section{Model}

The purpose of this study is to construct a detailed model of the CSE of HD 56126. In our modelling we focus on two key questions:

- The composition of the CSE, i.e. the amount of the various dust components present. As we have already outlined 
in Sect. 1 we observe emission from several components for which various carriers have been proposed. However, which of these components are present has not been firmly determined or the amounts of these carriers in the nebula have not been quantified.

- The location of the dust. The latter is important since there is considerable disagreement in the literature regarding the evidence for a superwind phase (with mass loss rates possibly exceeding $\left.10^{-4} M_{\odot} / y r\right)$. Meixner et al. (1997) and Dayal et al. (1998) describe the mass-loss history with a low massloss phase followed by a superwind phase. Recently, Kwok et al. (2002) have obtained high angular resolution mid-IR images and find no evidence for a large-scale sudden ejection of material. On the other hand, von Helden et al. (2000) have proposed that the " 21 " $\mu \mathrm{m}$ feature is carried by TiC clusters and that the formation of these clusters requires an short phase of extreme mass loss.

Note that the observed mid-IR emission is not spherically symmetric but shows three intensity maxima along the limbbrightened inner edge of the dust shell (Meixner et al. 1997; Dayal et al. 1998; Jura et al. 2000; Kwok et al. 2002) (see also Fig. 3). We ignore this non-sphericity in our model because doing so greatly reduces the number of free parameters without compromising our analysis of the dust composition and density as a function of distance from the star. Because the nebula is largely optically thin, the deviations from spherical symmetry do not influence our conclusions. To confine the radial extent of the dust we compare the model results with the azimuthal averaged intensity profiles derived from the mid-IR images.

To model the absorption and emission of the circumstellar dust we use the dust radiative transfer program MODUST. This code solves the radiative transfer equation in spherical geometry subject to the constraint of radiative equilibrium. This yields the temperature of the dust. The code allows for the presence of several different dust components of various grain sizes and shapes. The density of the grains as a function of distance can be prescribed in various ways. MODUST yields both the model SED and intensity maps. We refer to Bouwman et al. (2000) and Bouwman (2001) for a description of techniques used in MODUST. The following parameters for modelling the HD 56126 system are used:

- The input spectrum of the central star is given by a line blanketed Kurucz model atmosphere (Kurucz 1991). We use $T_{\text {eff }}=7250 \mathrm{~K}$ and $\log (g)=1.0$. With these parameters and the observed total integrated light of $33.6 \times 10^{-12} \mathrm{~W} / \mathrm{m}^{2}$ the radius and luminosity of the star are related to the distance by:

$R_{\star}=20.5 d$ and $L_{\star}=1051 d^{2}$,

where $R_{\star}$ is the stellar radius in units of solar radii, $L_{\star}$ is the stellar luminosity in units of solar luminosities and $d$ is the distance to the earth in kiloparsecs.

- The location of the dust is constrained by mid-IR imaging. The intensity maximum which is observed at $\sim 1.1^{\prime \prime}$ from the star corresponds to the inner edge of the dust shell in the case of optically thin emission, which is certainly satisfied at IR wavelengths. Taking into account the point-spread-function (PSF) of the telescope we find that the inner edge is located at $\sim 1.2^{\prime \prime}$. Because dust emission is clearly observed in all mid-IR images out to a $\sim 2.6^{\prime \prime}$ radius this constitutes a lower limit for the extent of the dust shell. However, the upper limit of the extent is not well constrained by current imaging and in the following we will treat the outer radius as a free parameter.

- The dust density $(\rho)$ as a function of distance from the star $(r)$ is specified in the form:

$\rho=\rho_{0}\left(\frac{r}{r_{0}}\right)^{-p}$,

where $\rho_{0}$ is the dust density at the location of the inner edge of the shell $\left(r_{0}\right)$ and the power $p$ determines the steepness of the density profile. For a time-independent mass loss $p=2$. If the mass-loss rate has increased over time $p>2$.

- The grain size distribution for each separate dust component can be specified with a power-law in the form:

$n(a) \propto a^{-q} ; \quad\left(a_{\min } \leq a \leq a_{\max }\right)$,

where $a$ is the grain radius, $n(a)$ it the number density of grains with radius $a, q$ is the power and $a_{\min }$ and $a_{\max }$ are the minimum and maximum grain radius, respectively.

- We choose to keep the dust composition constant as a function of distance from the star. This means that the relative fraction of each grain material component, specified by chemical composition and size is fixed throughout the nebula. The dust shell consists of separate grain populations, i.e. each dust grain contains one material, implying that separate species can have different temperatures at the same location.

\section{Optical properties}

In this section we discuss the optical properties of the various solid-state components that we use to model the HD 56126 system. Some of these materials have been well characterised in the laboratory over the broad wavelength range required for radiative transfer modelling. For other materials these data are not available and we describe how we treated the optical properties of these materials.

\subsection{Carbonaceous compounds}

There is general consensus that the mid-IR continuum in these sources is due to carbon-based dust grains, possibly with inclusion of hydrogen. The UIR bands at $~ 3.3,6-9$ and 10-17 $\mu \mathrm{m}$, indicated in Fig. 1 and 2 correspond to the vibrational resonances of aromatic and aliphatic $\mathrm{C}-\mathrm{H}$ and $\mathrm{C}-\mathrm{C}$ bonds. Despite this general consensus, the exact nature of the carrier is under much debate. This is partly due to the many different structures in which carbon can appear. There are three possible bonding types between the carbon-atoms: $\mathrm{sp}^{1}$, the aromatic $\mathrm{sp}^{2}$ bond, as in graphite, and the aliphatic $\mathrm{sp}^{3}$ bond, as in diamond. It is due to this diversity of bonding types of carbon and the resulting diversity in material properties of the various carbonaceous compounds that carbonaceous dust in space has evaded firm 
identification. In the following we consider amorphous carbon and hydrogenated amorphous carbon.

\subsubsection{Amorphous carbon}

The most prominent dust component that we observe is the mid-IR continuum. It carries $\sim 60$ per cent of the IR flux. This emission is generally attributed to amorphous carbon $(\mathrm{a}-\mathrm{C})$. We use the values of the complex refractive index of amorphous carbon as published by Preibisch et al. (1993). These data range in wavelength from 0.1 to $800 \mu \mathrm{m}$, which is sufficient to properly model the absorption and emission properties.

\subsubsection{Hydrogenated amorphous carbon}

The emission between 3-4 and $11-17 \mu \mathrm{m}$ is indicative of vibrationally excited $\mathrm{C}-\mathrm{H}$ bonds. This indicates that the CSE contains a compound of carbon and hydrogen. When looked at in detail both emission from $\mathrm{H}$-atoms bound to $\mathrm{sp}^{2}$ and $\mathrm{sp}^{3}$ sites is observed (Buss et al. 1990; Kwok et al. 2001). Such a mixture of hydrogen bonds is generally found in hydrogenated amorphous carbon (HAC). HAC is a likely condensate in $\mathrm{H}-$ rich and C-rich circumstellar ejecta. Therefore we include HAC in our model.

There is extensive literature on the properties of HAC in the material and surface science literature, where it is commonly referred to as a-C:H or diamond-like amorphous carbon. HAC films have received much attention as optical coatings because they can be manufactured to be relatively transparent in the 2-6 $\mu \mathrm{m}$ range; see Robertson (2002). As a result of this interest, such films have been studied in great detail: we refer to Robertson (2002) for an excellent review of HAC properties. In an astrophysical context, the properties of HAC have been extensively discussed by Duley and co-workers (e.g. Duley 1984; Scott et al. 1997; Grishko et al. 2001). Here we only discuss the optical properties relevant for the current study. Note that because of its application as optical coating the material research has been focused on those materials with low IR absorption. The more graphite-like materials, with high IR absorption levels (see below), have received much less attention.

HAC is a semiconductor. Its structure can be represented as $\mathrm{sp}^{2}$ bonded clusters surrounded by $\mathrm{sp}^{3}$ regions. The optical properties from the near-UV to the near-IR are determined by the $\pi$-electrons of the graphitic sp ${ }^{2}$ bonds. This is due to the fact that the $\pi$-electrons are weaker bound than the $\sigma$-electrons of the $\mathrm{sp}^{3}$ hybridisation. By varying the formation conditions, the $\mathrm{sp}^{2} / \mathrm{sp}^{3}$ ratio can be varied, which in turn allows to tune the absorptivity of HAC (Dischler et al. 1983). It is shown that the near-UV to visual absorption is determined by the size of the aromatic domains in the material (e.g. Angus et al. 1986; Robertson 1986, 2002). HAC contains a range of sizes of the aromatic domains and the level of absorption depends on the size of the largest aromatic clusters present in the structure at the concentration of a few per cent.
In the visual range, HAC absorptivity has an empirically determined dependence on photon energy and is usually expressed in the form of the so-called Tauc law (Tauc 1973):

$\alpha(E) E=A_{\mathrm{T}}\left(E-E_{\mathrm{T}}\right)^{2}$,

where $E$ is the photon energy of the incoming light, $\alpha(E)$ is the absorptivity as a function of energy, $A_{\mathrm{T}}$ is a constant that determines the slope and $E_{\mathrm{T}}$ is the Tauc gap. $E_{\mathrm{T}}$ and the optical band-gap (i.e. the energy level above which photons couple efficiently to the valence electrons) are directly linked to the average size of the $\mathrm{sp}^{2}$ domains.

At sub-band-gap energies the electronic absorptivity declines exponentially and is commonly expressed in an Urbach law:

$\alpha=\alpha_{\mathrm{U}} \exp \frac{E}{E_{\mathrm{U}}}$,

where $E_{\mathrm{U}}$ is the Urbach energy that determines the width of the tail, while $\alpha_{U}$ is a constant that determines the level. The width of the tail, i.e. $E_{\mathrm{U}}$, is determined by the disorder in the material. Hydrogenated amorphous carbon is a heterogeneous material with a range of bond types and sizes of $\mathrm{sp}^{2}$ bonded domains. As a consequence, the tail is broad. Typical values of the important parameters in the above equations are: $A_{\mathrm{T}} \simeq$ $300 \mathrm{~cm}^{-1} / \mathrm{eV}, E_{\mathrm{T}} \simeq 0-2 \mathrm{eV}$ and $E_{\mathrm{U}} \simeq 200 \mathrm{meV}$.

In the IR range, the contribution of the electrons to the absorptivity is in general small, unless one goes to materials with very large aromatic domains, the limiting case of which is the conductor graphite that exhibits a very strong electronic continuum in the IR. The IR absorption of HAC is dominated by vibrational resonances.

We combine the observed optical properties of different HAC materials from various authors in order to determine the dependence on structural parameters from the UV to the IR. We use the $\mathrm{H} / \mathrm{C}$ ratio as the parameter that determines the material properties. Note, that similar variations in the $\mathrm{sp}^{2} / \mathrm{sp}^{3} \mathrm{ra}-$ tio as described below are observed in pure amorphous carbon films (e.g. Robertson 2002). However, here we study the effect of $\mathrm{H} / \mathrm{C}$ because the astronomical spectra clearly show (through the features between 10 and $17 \mu \mathrm{m}$ ) that there are $\mathrm{H}$-atoms bound to the $\mathrm{C}$-atoms.

Compagnini et al. (1995) have studied the optical and IR absorptivity of HAC films as a function of hydrogen content. These authors list the parameters for both the Tauc law in the visible and the Urbach tail dominating the electronic continuum in the IR. For the HAC materials concerned here, hydrogen incorporation determines the structural parameters because hydrogen inclusion favours $\mathrm{sp}^{3}$ bonding. Hydrogen incorporation therefore limits the size of the graphitic domains. Thus, with increasing hydrogen content the electronic absorption decreases and the optical gap increases. This can be seen in Fig. 5, where we give the optical parameters of HAC as a function of hydrogen content.

A clear correlation is found between the optical gap and the strength of the integrated aromatic plus aliphatic $\mathrm{CH}$ stretching feature around $3.3 \mu \mathrm{m}$ (Dischler 1987). We use this relation to link the strength of the $\mathrm{CH}$ stretching mode to the electronic continuum level (cf. Fig. 5). 


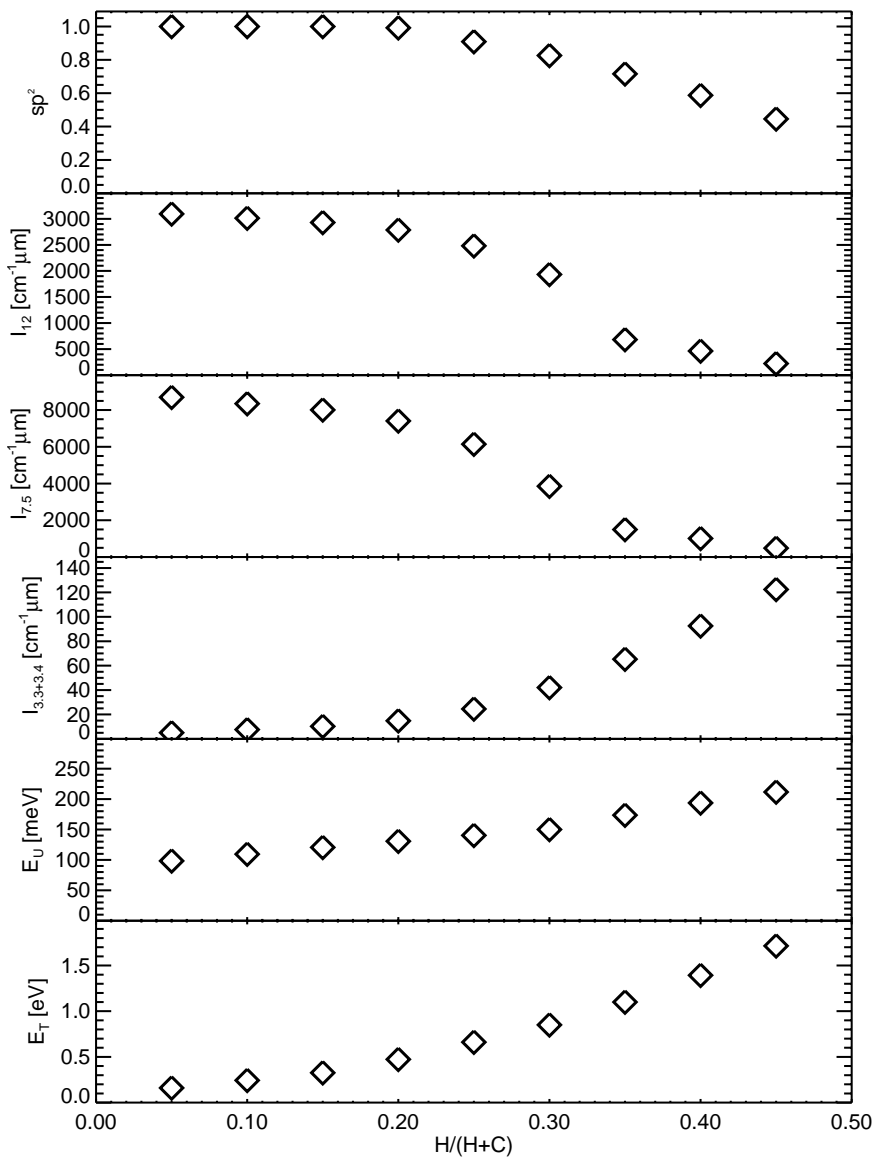

Fig. 5. The optical parameters of $\mathrm{HAC}$ as a function of $\mathrm{H}$ content. The panels show, from top to bottom: 1) the fraction of carbon bonded by $\mathrm{sp}^{2}$ bonds. 2), 3), 4) The integrated strength of the 11-17, 6-9 and the $3.3+3.4 \mu \mathrm{m}$ bands, respectively. 5) The Urbach energy (see Eq. (6)). 6) The Tauc energy (see Eq. (5)).

Bounouh et al. (1995) discuss the influence of annealing on the IR vibrational spectrum of HAC. Annealing removes H and as a result produces more and more graphite-like HAC. We use the strengths of the vibrational bands at 3.3, 3.4, 6-9 and $10-17 \mu \mathrm{m}$ for their series I material to empirically determine the strength of the bands as a function of $\mathrm{H} / \mathrm{C}$ ratio.

HAC material also exhibits a far-IR continuum due to vibrations. Unfortunately, these contributions have not been studied well because they contain little diagnostic value for the material properties. Following Schutte et al. (1993), we estimate the level of the far-IR vibrational continuum from the vibrational resonances observed in aromatic molecules. Moutou et al. (1996) have measured the far-IR spectra of a variety of different PAHs. We use the resonances these authors find to define an average far-IR continuum. We apply the following procedure to construct this average. We take the wavelength and absorption cross-section per C-atom for each of the 125 resonances listed in Table 3 of Moutou et al. (1996) and convolve these with a Gauss function with a full-width-at-half-maximum of $80 \mathrm{~cm}^{-1}$. After adding the contributions we renormalise by dividing by the total number of molecules (40) in the database and converting to absorptivity values in $\mathrm{cm}^{-1}$ using a typical value for the density of $2 \mathrm{~g} / \mathrm{cm}^{3}$. The far-IR contribution that

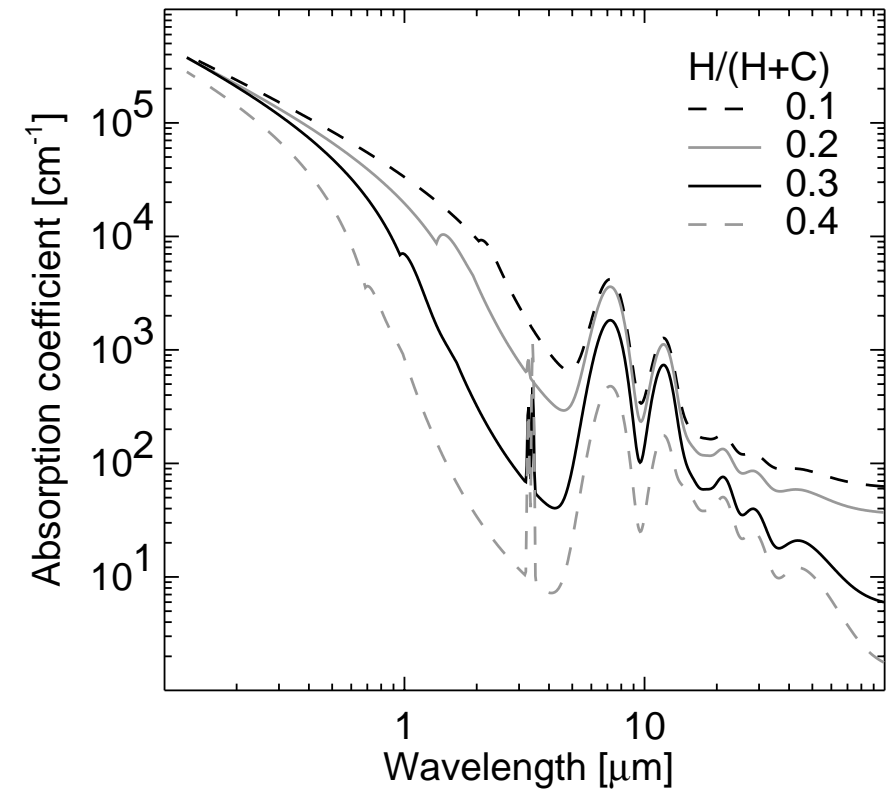

Fig. 6. Absorption coefficient $(\alpha)$ of HAC as a function of wavelength for changing values of hydrogen content. For details concerning the method used to derive the absorptivity, see text.

we obtain in this way is not completely smooth but shows some structure (see the 20-50 $\mu \mathrm{m}$ range in Fig. 6). This spectral structure is most likely due to the fact that we included a biased sample of "only" 40 molecules in a limited size range to construct the average absorption. A larger number of molecules with a wider range of molecular structures will probably fill in the gaps and yield a smoother far-IR continuum. Since this spectral structure is weak compared to the near- and mid-IR features and is lost in the emission from other dust components in the circumstellar spectrum, we have refrained from (arbitrarily) smoothing it out. The optical gap and the $\mathrm{sp}^{2}$ fraction are correlated as explained above. The measured relation for a large number of HAC and a-C materials is given in Robertson (2002, Fig. 58) from which we derive the $\mathrm{sp}^{2}$ fraction (cf. Fig. 5). The final estimate for the far-IR vibrational continuum is the product of the $\mathrm{sp}^{2}$ fraction and the average far-IR "continuum" as measured from PAHs. The estimate that we obtain is a lower limit to the real vibrational contribution to the far-IR absorptivity because we only consider the contributions due to the graphitic $\left(\mathrm{sp}^{2}\right)$ fraction in HAC, while in reality the $\mathrm{sp}^{3}$ skeleton will also contribute.

We show the dependence of the various parameters on the hydrogen incorporation in Fig. 5. The strong increase in the Tauc and Urbach energy with $\mathrm{H} /(\mathrm{H}+\mathrm{C})$ is apparent. Likewise, the 3.3 and $3.4 \mu \mathrm{m} \mathrm{CH}$ stretch modes increase with $\mathrm{H} /(\mathrm{H}+\mathrm{C})$ fraction. Notice how the strength of the $\mathrm{C}-\mathrm{H}$ out-of-plane bending modes between 11 and $17 \mu \mathrm{m}$ (in Fig. 5 represented by $I_{12}$ ) increases with decreasing $\mathrm{H}$ content. This seemingly contradictory behaviour is caused by the fact that in absolute terms the $\mathrm{H}$ bonded to $\mathrm{sp}^{2}$ sites increases during annealing. In this process some of the $\mathrm{H}$ bonded to the $\mathrm{sp}^{3}$ sites is converted into $\mathrm{H}$ bonded to $\mathrm{sp}^{2}$ sites. The absorptivity per $\mathrm{sp}^{2} \mathrm{C}-$ $\mathrm{H}$ bond is much larger than per $\mathrm{sp}^{3} \mathrm{C}-\mathrm{H}$ and the result is an 
increase of $I_{12}$. In contrast the aliphatic $\mathrm{CH}$ stretching modes around $3.4 \mu \mathrm{m}$ are stronger than the aromatic modes $(3.3 \mu \mathrm{m})$.

The wavelength dependent absorption cross-sections are shown in Fig. 6. The main effect is that the complete continuum level from the UV to the IR decreases with increasing hydrogen content. Because the UV/visible part of the spectrum decreases less than the IR, HAC with a higher $\mathrm{H}$ concentration will be warmer. While the continuum increases and the $\mathrm{CH}$ stretching modes at 3.3 strengthen with increasing $\mathrm{H}$ content, the mid-IR resonances between $6-9$ and $10-17 \mu \mathrm{m}$ diminish in relative strength. In the following we refer to $\mathrm{HAC}$ with $\mathrm{H} /(\mathrm{H}+\mathrm{C})=x$ as $\mathrm{HAC}_{x}$.

\subsection{Coal}

Guillois et al. (1996) have carried out a spectroscopic comparison between the emission features of carbon-rich post-AGBs and terrestrial coals. This comparison looks very promising. Of course, the CSE of post-AGB objects do not contain coals as such since coals are the product of the biological cycle in the earth-ecosystem. However, it may be worthwhile to consider materials with a very different formation mechanism as analogues to circumstellar materials.

In stark contrast to HAC, the optical properties of coal have been studied much less and no spectra that cover the full wavelength range from the UV to the IR for one single sample are available. Moreover there is a large spread in the derived complex refractive index of coals; see for example the comparisons made in Manickavasagam (1993) and Bhattacharya et al. (1996). This large spread is probably due to the wide variety of coal structural properties, even for materials that are labelled the same. When studying more controlled and limited samples of coal similar trends as described in Sect. 6.1.2 are recovered. For example, Ibarra et al. (1996) find a very similar dependence of the aromatic fraction on the hydrogen content as found in HAC. Likewise, similar behaviour for the strength of the 3.3 versus the $3.4 \mu \mathrm{m}$ band as a function of aromaticity (towards anthracite) is found (Černý 1996). Brewster \& Kunitomo (1984) find a significant increase in the free electron absorptivity upon graphitisation, again the same as is found in HAC. Unfortunately, there are no coal data available that cover the UV to the IR obtained using one single sample. However, from the similar behaviour found in coals and in HAC we conclude that our HAC parametrisation applies to coals as well.

One important difference between coals and HAC is the inclusion of atom impurities, primarily $\mathrm{O}$ and mineral phases in coals. Of course, our HAC model will not be able to reproduce the effects of these impurities. The effects of impurities in the CSE of carbon stars must however be limited because impurities have clear spectroscopic signatures like strong bands due to $\mathrm{O}-\mathrm{H}(\sim 3 \mu \mathrm{m})$ and $\mathrm{C}=\mathrm{O}$ groups $(5.8 \mu \mathrm{m})$ (Puente et al. 1998). Such signatures are not detected in the astronomical spectrum. We conclude that in so far coal can be used as an analogue for astronomical carbonaceous grains, its spectral properties can be well represented by those of HAC.

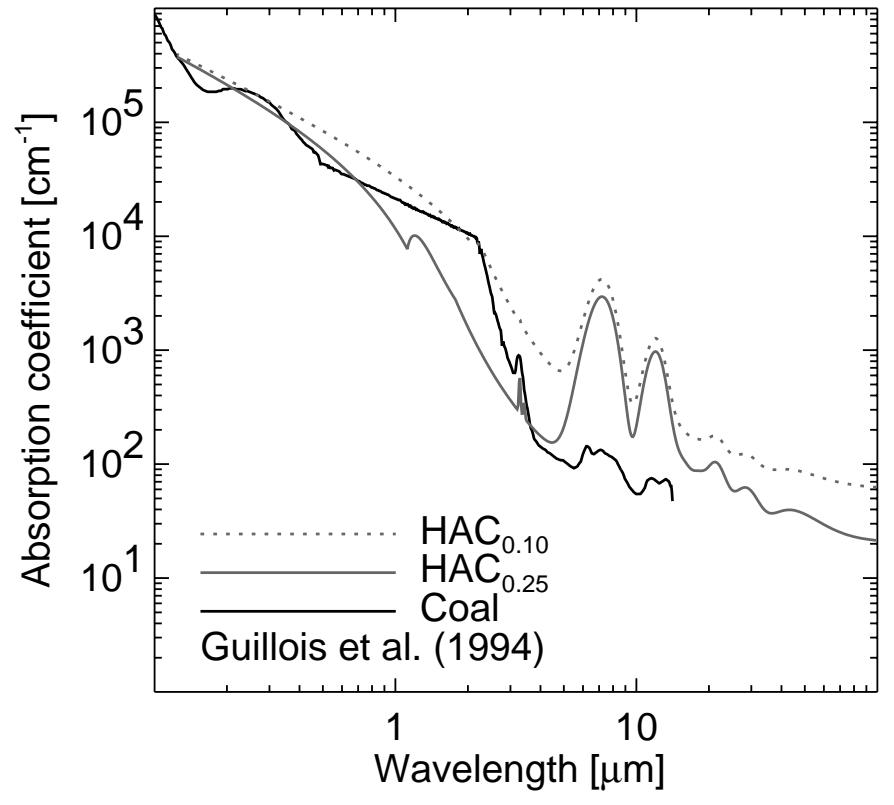

Fig. 7. A comparison between the optical properties of HAC as derived from our model and "standard coal" as taken from Guillois et al. (1994). The continuum of coal lies between the $\mathrm{H} /(\mathrm{H}+\mathrm{C})=0.10$ and the $\mathrm{H} /(\mathrm{H}+\mathrm{C})=0.25$ curves.

In Fig. 7 we show a comparison between the optical properties as derived from our model and the optical properties of "standard coal" taken from Guillois et al. (1994). Their coal optical properties are not derived from one single sample and the data from 0.7 to $3 \mu \mathrm{m}$ are not measured but interpolated. The interpolated optical properties in coal are higher than the Urbach tail in $\mathrm{HAC}_{0.25}$. At the same time, the IR absorption properties adopted by Guillois et al. (1994) are much weaker than the $\mathrm{HAC}_{0.25}$ features. This difference will give rise to a higher temperature for the "coal" than for HAC.

\subsection{Magnesium sulfide}

Begemann et al. (1994) have published the measured values of the complex refractive index ( $n$ and $k$ values) in the $10-500 \mu \mathrm{m}$ range of $\mathrm{Mg}_{x} \mathrm{Fe}_{(1-x)} \mathrm{S}$ with $x=0.0,0.1,0.5,0.75,0.9$. They find little difference between the samples with $x=0.75$ and $x=0.9$ and these authors suggest that the $x=0.9$ sample is a good substitute for $\mathrm{MgS}$. In order to reliably model the $\mathrm{MgS}$ contribution we need the optical properties in the UV to near-IR range as well. These data are unfortunately not available. We have opted to assume a grain size $\left(a_{\text {model }}\right)$ and calculate the mid-IR absorption cross section from the $n$ and $k$ values in a continuous distribution of ellipsoids (CDE) shape distribution following Bohren \& Huffman (1983, Chapter 12). We use the CDE distribution because this yields an emission profile closest to the observed profile in many carbon-rich evolved stars (e.g. Begemann et al. 1994; Szczerba et al. 1999), see Hony et al. (2002) for an elaborate discussion. By dividing the obtained cross section by the geometrical cross section we obtain the dimensionless absorption efficiency $Q(\lambda)$. In the wavelength range 0 to $1 \mu \mathrm{m}$ we assume $Q=1$, from 1 to $2 \mu \mathrm{m}$ we decrease $Q$ linearly to 0 and from 2 to $10 \mu \mathrm{m}$ we take $Q=0$. 
The assumptions above are of course unrealistic and will (most likely) overestimate the visual absorption for a given grain size. However, this does not influence the derived mass as we can easily derive the $\mathrm{MgS}$ grain temperature from the emission profile (see Hony et al. 2002). In thermal balance the $\mathrm{MgS}$ temperature is determined by the ratio of visual over the IR absorption efficiency. In general the IR absorption efficiency decreases more rapidly with decreasing grain size than the UV and visible absorption efficiency. Therefore, using the $\mathrm{MgS}$ temperature derived from the peak of the feature, there exists a grain radius $\left(a_{\mathrm{CSE}}\right)$ for which the absorption equals the observed emission in the MgS band. This value of $a_{\mathrm{CSE}}$ will be smaller or equal to the initial assumed grain size $a_{\text {model }}$. The mass that we derive does not depend on the grain size since the individual grains are optically thin at $30 \mu \mathrm{m}$ and therefore the emission scales with the mass (see also Sect. 7.4).

\subsection{Titanium carbide nano-crystals}

The identification of $\mathrm{TiC}$ as the carrier of the " 21 " $\mu \mathrm{m}$ feature is based on the wavelength coincidence of a strong resonance found in $\mathrm{TiC}$ nano-crystals with the " 21 " $\mu \mathrm{m}$ feature (von Helden et al. 2000). The optical properties of such nanocrystals have so far not been determined in the laboratory. The optical properties of TiC in the bulk have been studied extensively (Lye \& Logothetis 1966; Alward et al. 1975; Ihara et al. 1975; Lynch et al. 1980; Pflüger et al. 1984; Koide et al. 1990, 1993; Delin et al. 1996; Espinosa-Magaña et al. 2002). Although these studies are focused on the high energy range (the longest wavelength presented is $12 \mu \mathrm{m}$ ), they consistently find a large free electron contribution to the optical properties in the infrared. Henning \& Mutschke (2001) present the first midIR spectroscopy of bulk TiC. Their spectrum indeed shows a strong mid-IR continuum due to free electrons. They find no resonance at $20.1 \mu \mathrm{m}$ and only a very weak resonance near $19 \mu \mathrm{m}$, much weaker than the resonance found in nano-TiC.

In principle one can predict how some changes in spectral behaviour occur when comparing bulk optical properties with those of small ( $<200$ atoms) clusters (Kreibig \& Vollmer 1995). One of the effects is the limitation on the electron mean freepath (Kreibig \& Fragstein 1969). This effect is caused by "free" electrons that are scattered off the surface of the nano-crystal with a frequency that exceeds the frequency of collisions in the bulk material. In order to quantitatively calculate changes in the optical constants with respect to the bulk optical properties requires separating the contribution of non-conducting electrons to the optical absorption from the free-electron contribution. For example, a detailed description of how to apply this correction for silver is given in Alvarez et al. (1997). This separation is based on fitting the optical properties over a wavelength range where the free-electron contribution to the absorption provides the dominant source of opacity. However, in TiC there is no wavelength range were this is clearly the case. For example Koide et al. (1993) find (besides free-electron opacity) inter-band transitions, due to non-conducting electrons, up to $12 \mu \mathrm{m}$; the longest wavelength they measured. As a consequence, the estimated contribution due to free electrons derived in one wavelength range yields results that clearly over- or underestimate the free-electron contribution in other wavelength ranges.

Another observed effect is the strengthening of the $20 \mu \mathrm{m}$ resonance. If we translate the strength of the $20 \mu \mathrm{m}$ feature in nano-crystalline $\mathrm{TiC}$ to bulk samples this resonance would stand out clearly in the spectrum. Instead, a feature is barely detected (Henning \& Mutschke 2001). This immediately shows that bulk TiC cannot explain the observed " 21 " $\mu \mathrm{m}$ feature. Therefore, if TiC is the carrier of the " 21 " $\mu$ m feature the $\mathrm{TiC}$ must be in grains or in polycrystalline domains smaller than a few hundred atoms.

Other quantum effects may also be important in nanocrystalline materials. The localisation of the electrons may result in a material that behaves like a semi-conductor or insulator in the nano-crystalline phase even if the bulk material is a good conductor. Such behaviour has been observed for example in mercury (Busani et al. 1998).

The issues outlined above leave it impossible to derive reliable optical constants for $\mathrm{TiC}$ nano-crystals in a simple semi-empirical way. It is clear that these issues can only be satisfactorily resolved by laboratory measurements of the optical properties of nano-TiC. Still, for our radiative transfer calculations some assumptions about the optical properties of TiC must be made. For the emission of the $\mathrm{TiC}$ grains we have adopted an approach similar to the one outlined above for $\mathrm{MgS}$. We use the absorption profile and strength for TiC nanocrystals as presented by von Helden et al. (2000) and assume a constant level for the UV/visible absorption efficiency. This yields a TiC mass as a function of the UV/visible absorption level.

\section{Radiative transfer modelling}

\subsection{Initial model}

We first model only the dust continuum due to amorphous carbon. We use the parameters as described above. As a starting point for the fitting procedure we use a constant mass loss and outflow velocity profile $\left(\rho \propto r^{-2}\right)$ during a period of 10000 years. This corresponds to an envelope with radius of $\sim 10^{\prime \prime}$. We use a single grain size of $0.1 \mu \mathrm{m}$. Amorphous carbon grains of this size reach the highest temperature in radiative equilibrium, given the radiation field of the central star. We vary the density at the inner edge of the envelope $\left(\rho_{0}\right)$ to fit the emission at the shortest wavelengths of the SWS spectrum. The emission at the shortest wavelengths is sensitive only to the warmest dust which allows us to derive the mass of the warmest amorphous carbon grains. We choose 9-10 and $18 \mu \mathrm{m}$ as reference points. Below $9 \mu \mathrm{m}$ the data are noisy and/or the contributions from the star and the features are more difficult to estimate while at longer wavelengths the emission is partly due to cooler grains.

\subsection{Cold component/extent of the nebula}

The model that we construct in this way over predicts the flux levels beyond $40 \mu \mathrm{m}$ by $\sim 10-20$ percent, i.e. the model 
Table 4. HAC temperature and band strength as a function of $\mathrm{H} /(\mathrm{H}+\mathrm{C}) . \mathrm{H} / \mathrm{C}$ is the relative number of $\mathrm{H}$-atoms to $\mathrm{C}$-atoms. $T_{\max }$ is the maximum temperature of the grains. $F_{6-17, \mathrm{HAC}} / F_{6-17, \mathrm{obs}}$ is ratio of the integrated flux emitted in the plateau features in the model over the observed integrated flux in the plateau features, assuming equal mass of $\mathrm{HAC}_{X}$ and amorphous carbon.

\begin{tabular}{lccc}
\hline \hline & $\mathrm{H} / \mathrm{C}$ & $\begin{array}{c}T_{\max } \\
{[\mathrm{K}]}\end{array}$ & $F_{6-17, \mathrm{HAC}} / F_{6-17, \mathrm{obs}}$ \\
\hline $\mathrm{a}-\mathrm{C}$ & 0 & 170 & - \\
$\mathrm{HAC}_{0.10}$ & 0.11 & 188 & 1.4 \\
$\mathrm{HAC}_{0.20}$ & 0.25 & 190 & 1.7 \\
$\mathrm{HAC}_{0.30}$ & 0.43 & 195 & 1.4 \\
$\mathrm{HAC}_{0.35}$ & 0.54 & 205 & 0.8 \\
$\mathrm{HAC}_{0.40}$ & 0.67 & 205 & 0.5 \\
$\mathrm{HAC}_{0.45}$ & 0.82 & 205 & 0.2 \\
\hline
\end{tabular}

contains too much cold material. At the same time the modelled $11.9 \mu \mathrm{m}$ and $18.2 \mu \mathrm{m}$ spatial intensity profiles drop more steeply than observed within the closest 2.6". The same has been noted at shorter wavelengths by Meixner et al. (1997) and Jura et al. (2000). Meixner et al. (1997) use $\rho \propto r^{-1}(p=1)$ in order to fit their mid-IR images. However, this introduces even more cold dust located far from the star. We compare the observed $18.2 \mu \mathrm{m}$ intensity profile and the model profiles of the $p=1,2$ models in Fig. 10, below. The $p=2$ model peaks too much to the centre of the nebula and produces too little flux in the outer nebula, while the $p=1$ model is much closer to the observed intensity profile. Because the " $p=2$ "-model over predicts the flux levels at the longest wavelengths together with the fact that the observed intensity profile from the inner envelope $\left(1-2.6^{\prime \prime}\right)$ requires $p=1$, prompts us to limit the extent of the nebula.

From an observational point of view there is no evidence that the envelope extends beyond $\sim 2.6^{\prime \prime}$. All available images show only emission from this inner region. Note, that this is not conclusive evidence because the available mid-IR images are all at wavelength shorter than $25 \mu \mathrm{m}$, i.e. at the Wien side of the dust emission (see Fig. 1). This emission is extremely sensitive to the dust temperature and the cool dust far away from the star does not contribute much. Deep optical images that show scattered light from the nebula (Ueta et al. 2000) show no evidence for emission outside the $\sim 3^{\prime \prime}$ radius. Noteworthy in this respect is that the very high quality continuum images at 10 and $18 \mu \mathrm{m}$, as presented by Kwok et al. (2002), are very similar in morphology and size while a dust envelope that continues beyond $3^{\prime \prime}$ would cause the $18 \mu \mathrm{m}$ images to be $\sim 25$ per cent larger than the $10 \mu \mathrm{m}$ image, where the size is measured at 10 per cent of the peak intensity. This "growing" with increasing wavelength is not observed.

We construct a model with the same parameters as above but with $p=1$. This model fits the observed 11.9 and $18.2 \mu \mathrm{m}$ intensity profiles much better than a $r^{-2}$ law. From this we derive an upper limit on the outer radius of the envelope of 4 ". A larger envelope produces too much far-IR flux.
Up to now we only considered $0.1 \mu \mathrm{m}$ amorphous carbon grains. In reality grains of different sizes are expected to form (Dominik et al. 1989). We use a standard MRN size distribution (Mathis et al. 1977): $n(a) \propto a^{-3.5}$. We use $0.01 \mu \mathrm{m}$ and $1.0 \mu \mathrm{m}$ as the smallest and largest grain radius, respectively.

As described above the outer radius of the envelope is determined spectroscopically from the far-IR flux levels, i.e. the relative amounts of warm and cool a-C. A grain-size distribution affects these relative amounts because grains of different size reach different temperatures. Therefore, by using a grain-size distribution the outer radius can be further constrained. With the grain-size distribution as described we find that with a size of $4^{\prime \prime}$ the predicted far-IR fluxes are 50 per cent too high. We find that the best fitting outer radius of the envelope is $2.6^{\prime \prime}$. We use this outer radius in the following analysis.

\subsection{HAC temperature}

We include $\mathrm{HAC}$ as an additional component to a-C. We vary $\mathrm{H} / \mathrm{C}$ and the amount of HAC to best fit the observations. We do not aim to fit the features in detail but aim to determine if HAC reaches the temperature needed to explain the relative band strength of the broad emission features. With increasing $\mathrm{H} / \mathrm{C}$ both the UV/visible and the IR absorptivity decrease. Because the IR absorptivity decreases more steeply, grains with a high $\mathrm{H} / \mathrm{C}$ will be warmest. On the other hand, the amount of HAC required to explain the energy emitted in the features increases with $\mathrm{H} / \mathrm{C}$ content because $\mathrm{HAC}$ with high $\mathrm{H} / \mathrm{C}$ absorbs the stellar light less efficiently. In Table 4 we give the temperature that HAC grains reach at the inside of the envelope and the strength of the features as a function of hydrogen content. All HAC grains are warmer than a-C grains. In the $\mathrm{H} /(\mathrm{H}+\mathrm{C})=0.35-0.45$ range $\mathrm{HAC}$ grain reach a maximum temperature of $\sim 205$ K. $F_{6-17, \mathrm{HAC}} / F_{6-17, \text { obs }}$ gives the integrated strength of the plateau features relative to the observed strength assuming that the total HAC mass is equal to the a-C mass. Note, that we list the total flux emitted in the two plateau features. In all cases the flux emitted in the $10-17 \mu \mathrm{m}$ band dominates and the $6-9 \mu \mathrm{m}$ band is too weak compared to the observations, i.e. the temperature is too low.

As can be seen from Table $4, \mathrm{HAC}_{0.35}$ is optimal in the sense that it reaches a high temperature while requiring "only" equal mass as contained in a-C. To get the band strength of the 6-9 and 10-17 $\mu \mathrm{m}$ features right we would have to decrease the inner radius of the envelope. The band strengths are correct only when we would decrease the inner radius to $\sim 0.5^{\prime \prime}$. At $0.5^{\prime \prime}$ the HAC grains reach a temperature of $\sim 290 \mathrm{~K}$. However, such a small inner radius of the dust envelope is inconsistent with the available mid-IR images.

The comparison with the observations becomes worse in the $3 \mu \mathrm{m}$ region. In our model $\mathrm{HAC}_{0.35}$ produces no discernible aromatic $3.28 \mu \mathrm{m}$ or aliphatic $3.42 \mu \mathrm{m}$ feature while both are observed (Kwok et al. 1990). We conclude that HAC grains in radiative equilibrium do not reach the temperature required to explain the observed strength of the 3 and 6-9 $\mu \mathrm{m}$ features relative to the $10-17 \mu \mathrm{m}$ plateau features. 


\subsection{Magnesium sulfide}

Before we include $\mathrm{MgS}$ in our model we derive the mass of the $\mathrm{MgS}$ component in a simple, relatively model independent, way. In the limit of grains much smaller than the wavelength (Rayleigh limit), the absorptivity of a grain scales linearly with the mass of the grain. In the optically thin case the observed flux can thus be expressed in terms of distance from the earth $(d)$, grain temperature $(T)$ and total mass $(M)$ alone. This can easily be derived in the following way. Consider the flux density emitted by a single grain $\left(f_{1}\right)$ of radius $a$ and temperature $T$ :

$$
\begin{aligned}
f_{1}(\lambda, a, T(a)) & =4 \pi a^{2} Q(\lambda, a) \pi B(\lambda, T(a)) \\
& =3 V(a) \frac{Q(\lambda, a)}{a} \pi B(\lambda, T(a)),
\end{aligned}
$$

where $\lambda$ is the wavelength, $Q(\lambda, a)$ is the absorption efficiency; thus $4 \pi a^{2} Q(\lambda, a)$ is the effective radiating surface of the grain, $\pi B(\lambda, T(a))$ is the black body flux density emitted per unit area and $V(a)$ is the volume of the grain. In the Rayleigh limit $\frac{Q(\lambda, a)}{a}$ is independent of $a$ and therefore $T$ is independent of $a$ and we can write:

$$
\begin{aligned}
f_{1}(\lambda, a, T) & =3 \frac{M(a)}{\rho} C(\lambda) \pi B(\lambda, T) \Rightarrow \\
f_{\mathrm{tot}}(\lambda, T) & =\sum_{i} 3 \frac{M\left(a_{i}\right)}{\rho} C(\lambda) \pi B(\lambda, T) \\
& =3 \frac{M_{\mathrm{tot}}}{\rho} C(\lambda) \pi B(\lambda, T),
\end{aligned}
$$

where $M(a)$ is the mass of the grain, $\rho$ is the density of the material $C(\lambda)=\frac{Q(\lambda, a)}{a}$ and $f_{\text {tot }}$ is the total flux density emitted by all grains, the summation is done over all grains $(i)$ and $M_{\text {tot }}$ is the total mass of the grains. Integrating over wavelength and correcting for the distance we finally obtain:

$$
\begin{aligned}
F_{\lambda_{0,1}} & =\frac{1}{4 \pi d^{2}} \int_{\lambda_{0}}^{\lambda_{1}} 3 \frac{M_{\mathrm{tot}}}{\rho} C(\lambda) \pi B(\lambda, T) \mathrm{d} \lambda \quad \text { and } \\
M_{\mathrm{tot}} & =\frac{4}{3} F_{\lambda_{0,1}} d^{2} \rho\left(\int_{\lambda_{0}}^{\lambda_{1}} C(\lambda) B(\lambda, T) \mathrm{d} \lambda\right)^{-1},
\end{aligned}
$$

where $F_{\lambda_{0,1}}$ is the observed flux integrated between $\lambda=\lambda_{0}$ and $\lambda=\lambda_{1}$. The last term in Eq. (9) depends only on $T$ for a given material. The peak position of the " 30 " $\mu \mathrm{m}$ feature is a good indicator of $T$ for MgS (Hony et al. 2002) and we derive $T_{\mathrm{MgS}} \simeq 150 \mathrm{~K}$ from the observed peak position in the spectrum of HD 56126. Using the measured values $\rho=3 \mathrm{~g} / \mathrm{cm}^{3}, F_{10,50}=2.7 \times 10^{-12} \mathrm{~W} / \mathrm{m}^{2}$ and $d=2400 \mathrm{pc}$ we find $M_{\mathrm{MgS}}=9 \times 10^{-6} M_{\odot}$.

If we assume that all $\mathrm{S}$ is condensed in the form of $\mathrm{MgS}$ we can calculate the total corresponding envelope mass $\left(M_{\text {env }}\right)$. Van Winckel \& Reyniers (2000) have measured the atmospheric abundance by number of both $\mathrm{Mg} / \mathrm{H}$ and $\mathrm{S} / \mathrm{H}$ to be $\sim 4 \times 10^{-6}$, which yields a $M_{\text {env }}$ of $0.06 M_{\odot}$. The mass of the envelope is variously estimated by fitting the SED and mid-IR imaging at $0.54 M_{\odot}$ (Meixner et al. 1997), $0.26 M_{\odot}$ (Dayal et al. 1998) and $0.4 M_{\odot}$ that we derive below. We conclude that the abundances of $\mathrm{Mg}$ and $\mathrm{S}$ are consistent with the strength of the " 30 " $\mu \mathrm{m}$ feature.

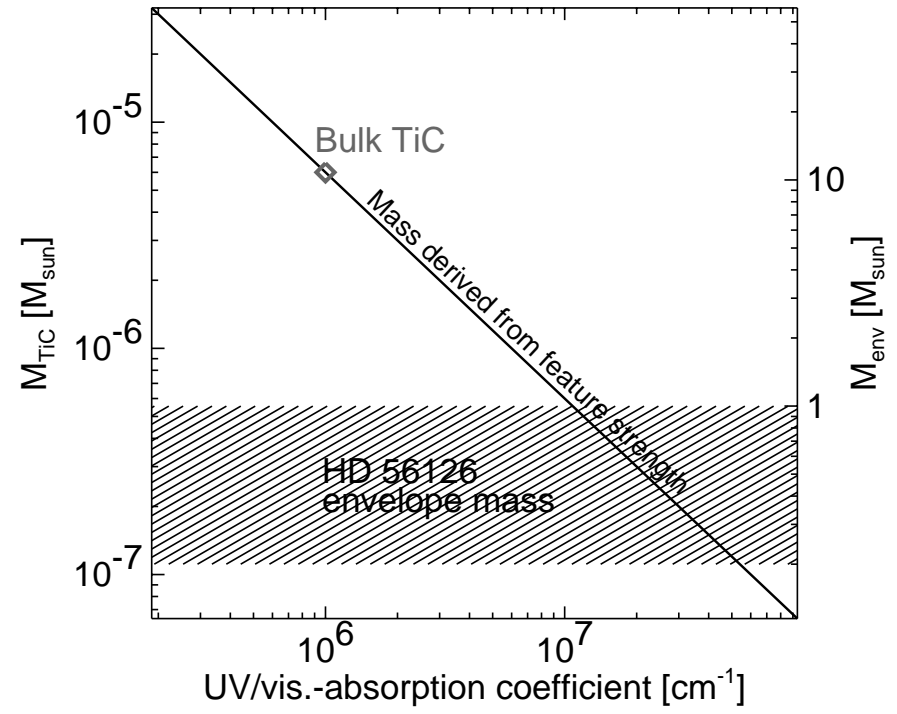

Fig. 8. The mass of the TiC component as a function of the UV/visible absorption coefficient $(\alpha)$. We show the TiC mass derived from the strength of the " 21 " $\mu \mathrm{m}$ feature for different values of the UV/visible absorption coefficient (black line). A higher absorption coefficient requires less TiC. The grey diamond indicates the measured absorption coefficient of bulk TiC. The right abscissa shows the corresponding total envelope mass. The hatched area indicates reasonable values for the total envelope mass. The absorption coefficient of nano-TiC needs to be $\sim 20$ times higher that bulk to be compatible with the observed strength of the " 21 " $\mu \mathrm{m}$ feature.

Of course, these values of $M_{\mathrm{MgS}}$ and $M_{\mathrm{env}}$ are lower limits. First, because we have assumed that all the $\mathrm{MgS}$ is at $150 \mathrm{~K}$. In reality there will be a range of temperatures. The colder grains are more "hidden" and this increases $M_{\mathrm{Mgs}}$, see Eq. (9). However, while such a temperature is too low to be compatible with the observed peak position of the " 30 " $\mu \mathrm{m}$ feature, decreasing $T_{\mathrm{MgS}}$ to $100 \mathrm{~K}$ increases $M_{\mathrm{MgS}}$ only by a factor 5 , which still yields a reasonable value for $M_{\text {env }}$. Second, because we assume that all $\mathrm{Mg}$ - and S-atoms are condensed into $\mathrm{MgS}$ to derive $M_{\text {env }}$ from $M_{\mathrm{Mgs}}$. We point out that there are no other $\mathrm{Mg}$ or $\mathrm{S}$ bearing components identified in the nebula. We conclude that from a simple analysis the strength of the $\mathrm{MgS}$ feature as observed in HD 56126 is compatible with the available Mgand S-atoms.

We include $\mathrm{MgS}$ in the radiative transfer model using the optical properties as described in Sect. 6.3. The only free parameters are the $\mathrm{MgS}$ grain size and the relative mass fraction. We find that a grain size of $a_{\mathrm{MgS}} \simeq 0.01-0.02 \mu \mathrm{m}$ fits best with the observed profile of the " 30 " $\mu \mathrm{m}$ feature. Larger grains are too cold and cause the " 30 " $\mu \mathrm{m}$ feature to peak at longer wavelength than observed. The mass of the $\mathrm{MgS}$ component is 4 per cent of the a-C, or $1.4 \times 10^{-5} M_{\odot}$ at $d=2.4 \mathrm{kpc}$; this is in good agreement with the $9 \times 10^{-6} M_{\odot}$ we derive from the simple considerations above, assuming $T_{\mathrm{MgS}}=150 \mathrm{~K}$.

\subsection{Titanium carbide}

As discussed above (Sect. 6.4) only TiC nano-crystals are expected to show a strong " 21 " $\mu \mathrm{m}$ feature. Such nano-crystals 


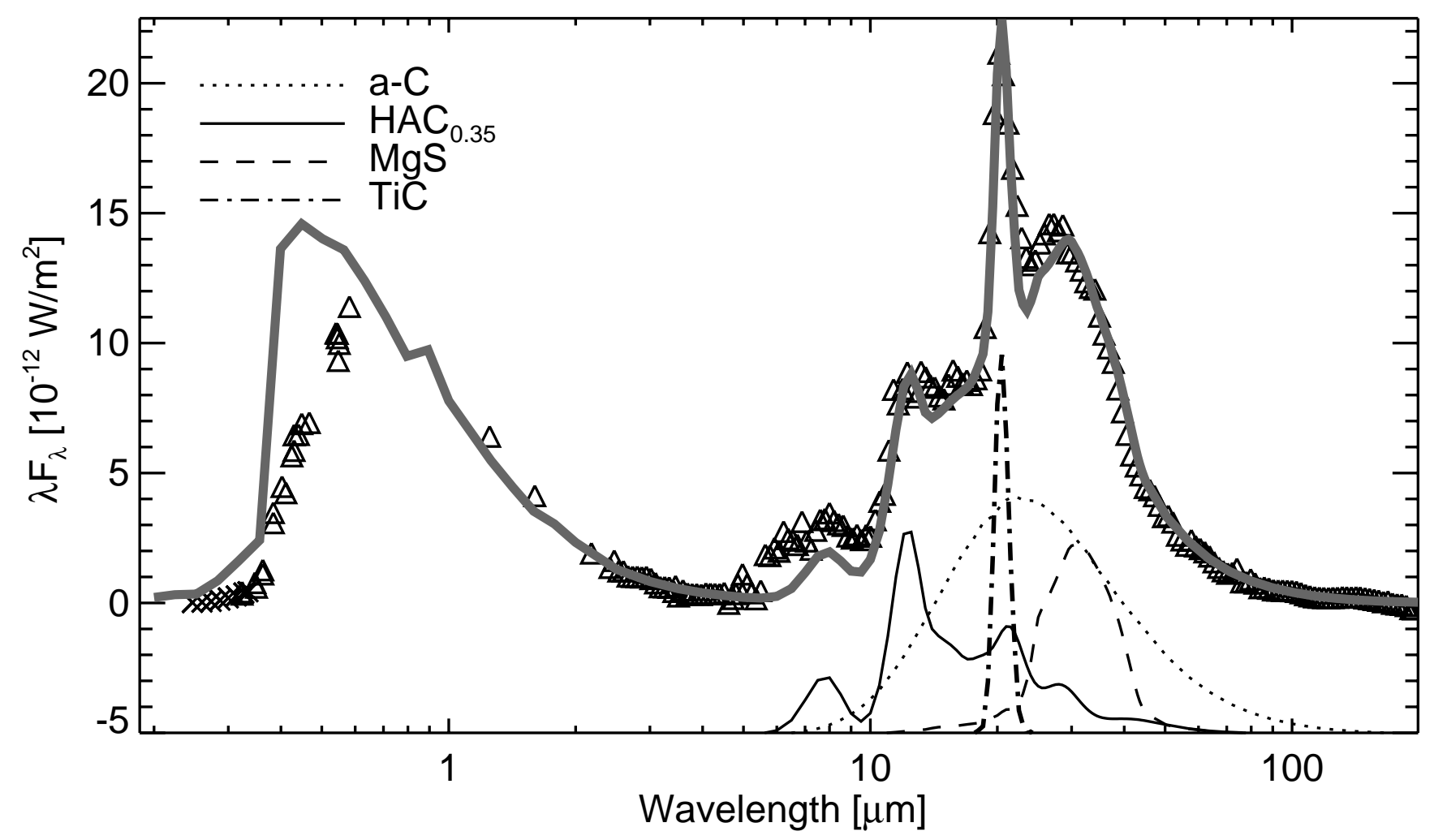

Fig. 9. A comparison between the observed SED (triangles) and our best fitting model (thick grey line). The model reproduces the dust continuum and the " 30 " $\mu \mathrm{m}$ feature very well. The model does not reproduce the strength of the $6-9 \mu \mathrm{m}$ feature and the extinction in the UV. Underneath the IR emission spectrum we give an indication of the contributions of the various dust components offset by $-5 \times 10^{-12} \mathrm{~W} / \mathrm{m}^{2}$.

will undergo a temperature spike upon absorption of a single UV/visible photon. Cooling then occurs through emission in the " 21 " $\mu \mathrm{m}$ feature. The mass of $\mathrm{TiC}$ is directly determined from the energy balance: the IR flux is equal to the absorbed $\mathrm{UV} /$ visible flux, which is given by our radiative transfer calculation. In Fig. 8 we show the mass of TiC in the envelope that we derive as a function of the UV/visible absorption coefficient using the Ti/H abundance of $1.3 \times 10^{-8}$ (van Winckel $\&$ Reyniers 2000). We also show the measured absorption coefficient of bulk TiC. It is clear from Fig. 8 that bulk TiC can not explain the strength of the feature even if all the energy absorbed would be emitted solely in the " 21 " $\mu$ m resonance. In order to be compatible with the observed band strength nanoTiC needs to absorb 20 times more efficiently per Ti-atom in the $0.2-0.7 \mu \mathrm{m}$ wavelength range than bulk TiC. This corresponds to a visual absorption efficiency $\left(Q_{\text {vis }}\right)$ of $\sim 8$ for a $10 \times 10 \times 10$ atom nano-crystal. Such a high value for the visual absorption coefficient is only possible if, due to the nanometer size scale, a strong electronic resonance is present in nano-TiC. This problem is already evident in the complete spectrum as presented in Fig. 1. Either the carrier of the " 21 " $\mu \mathrm{m}$ feature is as abundant as $\mathrm{MgS}$ or it has a much higher UV/visible absorptivity.

\section{Results and discussion}

\subsection{Best fit model}

In Fig. 9 we compare the predicted and observed SED. Our best model fits the observations in many details. Note that the ordinate scale is linear while previously published SED fits are compared on a logarithmic basis. The peak of the SED, the shape and strength of the " 30 " $\mu \mathrm{m}$ feature and the shape of the " 21 " $\mu \mathrm{m}$ feature are very well explained by the model. There are two places where the model fails to reproduce the details of the observations: i) The UV flux levels are over predicted. It is clear that an important UV absorption component is missing in our model. Laboratory studies of the UV absorption properties of $\mathrm{MgS}$ and $\mathrm{TiC}$ are therefore needed. ii) The strength of the $6-9 \mu \mathrm{m}$ band is too small in the model. The discrepancy at $6-9 \mu \mathrm{m}$ is discussed in Sect. 8.4.

In Fig. 10 we show the comparison between the observed and predicted intensity profiles. The strength and position of the intensity maximum and width of the nebula are reproduced very satisfactorily by the $p=1$ model. In the outer parts of the nebula this model under predicts the intensities slightly. This may be due to deviations of the true PSF from the assumed Gaussian PSF, some weak background in the observation or to weak nebular emission that we do not include in the model. The best fitting $p=2$ model produces an intensity profile that is too much peaked towards the inner edge of the dust shell.

Towards the centre of the circumstellar shell there is a rise in the observed intensity, which is present in all available midIR images. At $18.2 \mu \mathrm{m}$, this central region contains about $3 \mathrm{Jy}$ which is $\sim 100$ times more than the photospheric contribution of the central star at $18.2 \mu \mathrm{m}$. Therefore, this rise must be due to the departure from spherical symmetry of the nebula, causing more dust to be present along the line of sight towards the 


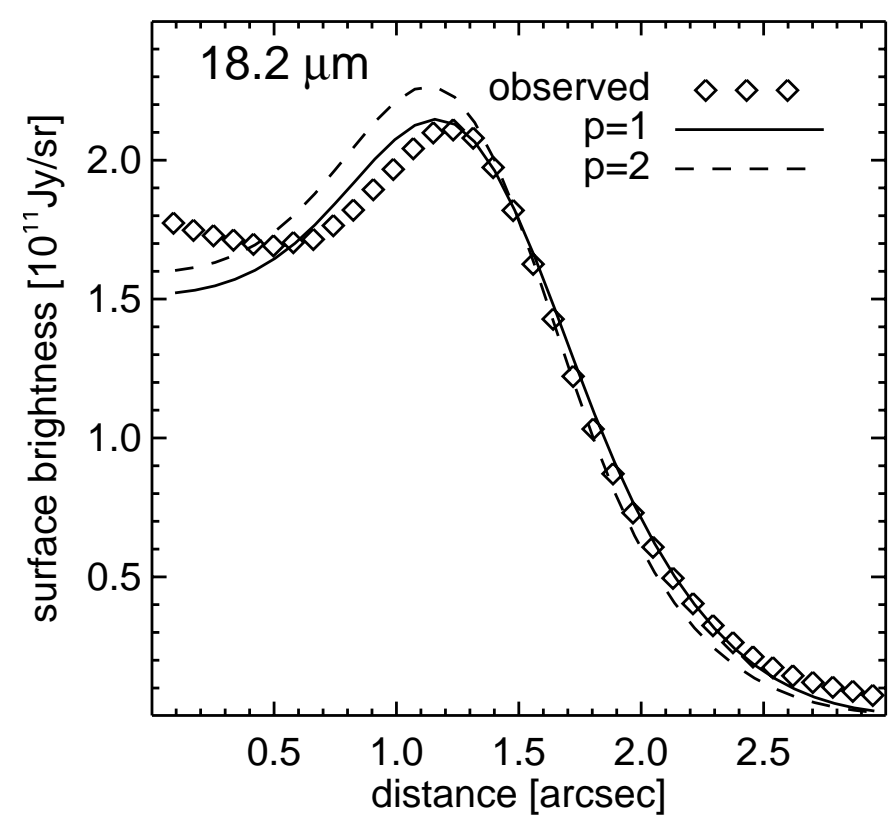

Fig. 10. A comparison between the observed intensity profile (diamonds) as a function of distance from the centre of the nebula, the intensity profile from the best fitting radiative transfer model (line) and the profile predicted the $p=2$ model (dashed line). The observed profile is obtained by azimuthal averaging of the $18 \mu \mathrm{m}$ image as published by Kwok et al. (2002). The model profiles are obtained by averaging the intensity profile of the model shown in Fig. 9 and the $p=2$ model after convolution with a 2D-Gaussian PSF with the same fullwidth-at-half-maximum as the $18 \mu \mathrm{m}$ observation.

central star or it is due to an additional dust component which is not present in our model. The nature and origin of such an additional dust component is at present unknown.

We note that the emission from this region, which is possibly located closer to the central star than the inner edge of the dust shell in our model, cannot explain the plateau features and the high temperatures they require (Sect. 7.3), because the flux contributions from this region are much too small and the plateau features are emitted from a much more extended region, e.g. Fig. 3 and the images presented in Meixner et al. (1997); Dayal et al. (1998); Jura et al. (2000); Kwok et al. (2002)

We also note that our model fails to reproduce the observed intensity profiles at shorter wavelengths (10.3, 11.7 and $12.5 \mu \mathrm{m}$ ). The observed profiles are systematically broader than predicted. This is an indirect but clear indication that the carriers of the plateau emission features at those wavelengths are not in radiative equilibrium, but are transiently heated to temperatures higher than expected on the basis of our equilibrium model.

In Table 5 we summarise the main parameters of the best fit model. We find that the observations are best explained with a compact dust shell that extents from 1.2-2.6". The mass of the dust contained in the shell is $\sim 7 \times 10^{-4} M_{\odot}$. Assuming a constant outflow velocity of $10.7 \mathrm{~km} \mathrm{~s}^{-1}$ (Knapp et al. 1998) we calculate that the mass-loss commenced $2800 \mathrm{yr}$ ago and halted $1300 \mathrm{yr}$ ago. Using a value of 220, as is often used for carbon-rich circumstellar matter, for $M_{\text {gas }} / M_{\text {dust }}$ we find that the time averaged mass-loss rate is $\sim 10^{-4} M_{\odot} /$ yr. As argued
Table 5. The best fitting model: parameters and derived quantities.

\begin{tabular}{|c|c|c|c|c|c|}
\hline \multicolumn{5}{|c|}{ Central star } & \multirow[t]{5}{*}{ Comments } \\
\hline$d$ & & {$[\mathrm{kpc}]$} & 2.4 & & \\
\hline$T_{\text {eff }}$ & & {$[\mathrm{K}]$} & 7250 & & \\
\hline$R_{\star}$ & $\propto d^{2}$ & {$\left[R_{\odot}\right]$} & 49 & & \\
\hline$L_{\star}$ & $\propto d^{2}$ & {$\left[L_{\odot}\right]$} & 6000 & & \\
\hline \multicolumn{6}{|c|}{ Dust shell } \\
\hline$R_{\text {in }}$ & $\propto d$ & {$[\mathrm{~cm}]$} & $4.5 \times 10^{16}$ & & $1.2^{\prime \prime}$ \\
\hline$R_{\text {out }}$ & $\propto d$ & {$[\mathrm{~cm}]$} & $9.3 \times 10^{16}$ & & $2.6^{\prime \prime}$ \\
\hline$v_{\text {exp }}$ & & {$\left[\mathrm{km} \mathrm{s}^{-1}\right]$} & 10.7 & & Knapp et al. (1998) \\
\hline$\Delta t_{\text {in }}$ & $\propto d / v_{\exp }$ & {$[\mathrm{yr}]$} & 1300 & & \\
\hline$\Delta t_{\text {out }}$ & $\propto d / v_{\exp }$ & [yr] & 2800 & & \\
\hline$\rho_{0}$ & & {$\left[\mathrm{~g} / \mathrm{cm}^{3}\right]$} & $8 \times 10^{-22}$ & & \\
\hline$p$ & & & 1 & & $\rho(r)=\rho_{0}\left(\frac{r}{R_{\text {in }}}\right)^{-p}$ \\
\hline \multicolumn{2}{|c|}{ Dust species } & & Mass & $\%$ & \\
\hline dust & $\propto d^{2}$ & {$\left[M_{\odot}\right]$} & $7.4 \times 10^{-4}$ & 100 & \\
\hline $\mathrm{a}-\mathrm{C}$ & $\propto d^{2}$ & {$\left[M_{\odot}\right]$} & $3.6 \times 10^{-4}$ & 49 & $a=0.01-1 \mu \mathrm{m}$ \\
\hline HAC & $\propto d^{2}$ & {$\left[M_{\odot}\right]$} & $3.6 \times 10^{-4}$ & 49 & $\mathrm{H} /(\mathrm{H}+\mathrm{C})=0.35$ \\
\hline $\mathrm{MgS}$ & $\propto d^{2}$ & {$\left[M_{\odot}\right]$} & $1.4 \times 10^{-5}$ & 2 & $\mathrm{CDE}$ \\
\hline $\mathrm{TiC}$ & $\propto d^{2}$ & {$\left[M_{\odot}\right]$} & $8 \times 10^{-6}$ & - & $Q_{\text {vis }}=Q_{\text {bulk }}$ \\
\hline $\mathrm{TiC}$ & $\propto d^{2}$ & {$\left[M_{\odot}\right]$} & $3 \times 10^{-7}$ & - & $Q_{\mathrm{vis}}=10$ \\
\hline
\end{tabular}

Envelope

\begin{tabular}{lllcc}
\hline $\begin{array}{l}M_{\text {env }} \\
<\dot{M}>d^{2}\end{array}$ & $\propto d v_{\text {exp }}$ & $\begin{array}{l}{\left[M_{\odot}\right]} \\
{\left[M_{\odot} / \mathrm{yr}\right]}\end{array}$ & $\begin{array}{c}0.16 \\
1 \times 10^{-4}\end{array}$ & $\begin{array}{c}M_{\text {gas }} / M_{\text {dust }}=220 \\
M_{\text {gas }} / M_{\text {dust }}=220\end{array}$ \\
\hline & & $M_{\text {gas }} / M_{\text {dust }}=600$, see Sect. 8.3 \\
\hline$M_{\text {env }}$ & $\propto d^{2}$ & {$\left[M_{\odot}\right]$} & 0.44 & $M_{\text {gas }} / M_{\text {dust }}=600$ \\
$<\dot{M}>$ & $\propto d v_{\text {exp }}$ & {$\left[M_{\odot} / \mathrm{yr}\right]$} & $3 \times 10^{-4}$ & $M_{\text {gas }} / M_{\text {dust }}=600$ \\
\hline
\end{tabular}

below, a value of 600 for $M_{\text {gas }} / M_{\text {dust }}$ is more appropriate for this low metallicity object, corresponding to a mass-loss rate of $\sim 3 \times 10^{-4} M_{\odot}$. We find that 2 per cent of the dust (by mass) is contained in $\mathrm{MgS}$, consistent with the observed photospheric abundance of $\mathrm{Mg}$ and $\mathrm{S}$.

\subsection{Previous dust models}

The circumstellar environment of HD 56126 has been studied before using both mid-IR spectroscopic and imaging observations (Meixner et al. 1997; Dayal et al. 1998; Hrivnak et al. 2000; Jura et al. 2000). It is useful to compare the parameters we derive to those found before. One main difference between our model and previous work is the fact that we treat the dust composition in considerable detail. However, there are other significant differences as well. We find that the CSE of HD 56126 is well represented by a compact shell of 2.6" radius. We have argued above that a larger outer radius will cause an increase of the size of the nebula with increasing wavelength, contrary to the observations. Of course, a preceding phase with a much lower mass-loss rate would go undetected. The model of Skinner et al. (1995) contains a more extended component. The absence of this more extended component in our model is reflected in relatively low envelope mass 
of $0.16 M_{\odot}$ that we derive. This is because cold material far away does not contribute to the mid-IR fluxes but does contain mass. This "hidden" component is therefore important in determining the total envelope mass. Imaging at sub-mm wavelengths is needed to better constrain the cold envelope mass.

Skinner et al. (1995) find $M_{\text {env }}=0.54 M_{\odot}$ at a distance of $3 \mathrm{kpc}$, with two-thirds of the mass in the extended component. The mass of the inner component that they derive corresponds well to the mass of the a-C grains that we derive after correcting for the distance. Dayal et al. (1998) derive an envelope mass of $0.26 M_{\odot}$ at $2.7 \mathrm{kpc}$, which corresponds to $0.2 M_{\odot}$ at $2.4 \mathrm{kpc}$. These authors use a compact model with all dust contained within $2.2^{\prime \prime}$ but with two distinct fixed temperatures. In view of the difference in approach, the envelope mass they find and our mass determination are compatible. Hrivnak et al. (2000) derive a mass-loss rate of $3.6 \times 10^{-5} M_{\odot} / \mathrm{yr}$ at the distance of $2 \mathrm{kpc}$, which corresponds to $5 \times 10^{-5} M_{\odot} / \mathrm{yr}$ at $2.5 \mathrm{kpc}$, about half of the value that we find. Jura et al. (2000) model the density in the envelope as the result of a "dying" wind, i.e. a decreasing mass-loss rate with time. This is qualitatively consistent with the $\rho_{0}\left(r / R_{\text {in }}\right)^{-1}$ density prescription that we use. These authors find a maximum mass-loss rate of $\sim 3 \times 10^{-5} M_{\odot} / y$. However, they model the emission of the dust with a simplified amorphous carbon opacity function. This yields a mass estimate compatible with the warmest a-C grains ( $a=0.1 \mu \mathrm{m}$ ) in our model. Correcting for the mass of the other grains, the different distance and the $M_{\text {gas }} / M_{\text {dust }}$ ratio they use, they find $1.1 \times 10^{-4} M_{\odot} / \mathrm{yr}$ compatible with the mass-loss rate that we derived.

\subsection{Envelope mass; $M_{\text {gas }} / M_{\text {dust }}$}

The total envelope mass that we and other authors derive scales with the gas-to-dust mass ratio. For carbon-rich AGB and postAGB stars values between 200 and 250 are often used (e.g. Jura 1986; Meixner et al. 1997) and in the above derivations we have used 220. However this value is very uncertain. Because the bulk of the dust is carbon-based one can expect $M_{\text {gas }} / M_{\text {dust }}$ to strongly depend on the $\mathrm{C} / \mathrm{O}$ ratio. The photospheric abundances determination of van Winckel \& Reyniers (2000) yield $[\mathrm{C} / \mathrm{H}]=8.53-8.77$ and $\mathrm{C} / \mathrm{O}=0.7-1.4$. Taking values in the high end of this range and assuming that all carbon is locked in either CO (70 per cent) or dust (30 per cent) we find $M_{\text {gas }} / M_{\text {dust, } \mathrm{C}} \gtrsim 600$, in which $M_{\text {dust, } \mathrm{C}}$ is the mass of the carbon-based dust component. This high value of $M_{\text {gas }} / M_{\text {dust }}$ follows directly from the elemental abundance measurements. We find $M_{\text {dust,C }}=7.4 \times 10^{-4} M_{\odot}$ (see Table 5) which corresponds to $M_{\text {gas }}=0.44 M_{\odot}$. When we consider that the star had a ZAMS-mass of $\sim 1.1 M_{\odot}$ and the core-mass is roughly $0.6 M_{\odot}$ we conclude that nearly the entire envelope was lost during a high mass loss phase of $\sim 1500 \mathrm{yr}$. We also conclude that only a small amount of the dust can be "hidden" in cold grains, either far away from the star or in larger grains. This argues strongly against the importance of grey extinction due to large grains. Using the envelope mass of $0.44 M_{\odot}$ we find a high time-averaged mass-loss rate of a $\sim 3 \times 10^{-4} M_{\odot}$. We conclude that $H D 56126$ has experienced a short period (1500 year) of very strong mass loss $\left(3 \times 10^{-4} M_{\odot} / y r\right)$ during which the entire envelope was lost. If we adopt lower $\mathrm{C} / \mathrm{H}$ and $\mathrm{C} / \mathrm{O}$ values this conclusion would only be strengthened.

\subsection{The temperature of the $H A C$}

One of the main discussion points concerning the relation between HAC (or any other solid carrier) and the 3 to $17 \mu \mathrm{m}$ features found in C-rich environments is whether a solid particle in radiative equilibrium is able to explain the observed spectra. In particular the large feature to continuum ratios found in the astronomical spectra and the relatively short wavelengths ( $3 \mu \mathrm{m})$ at which emission is found appear difficult to obtain in radiative equilibrium. Indeed, one can immediately see from Fig. 1 precisely that difficulty; the peak of the SED lies beyond $20 \mu \mathrm{m}$ while strong features on a weak continuum are present in the 5-17 $\mu \mathrm{m}$ range. The fundamental question is whether solid grains can reach the temperature required to emit efficiently at these wavelengths.

We have tested this by building a detailed model of the optical properties of HAC. We find that in radiative equilibrium HAC does not reach a high enough temperature at the location of the dust. We have tested this too for "coal" with the opacities as published by Guillois et al. (1994), even though the main difference with HAC is due to the interpolation these authors applied. We find that their "coal" reaches a maximum temperature of $\sim 350 \mathrm{~K}$ at the inner edge of the dust shell. Which is not high enough to explain the relative band-strength of the plateau features $(\sim 500-600 \mathrm{~K})$.

This implies that the carriers that give rise to the plateau features are not in radiative equilibrium with the stellar radiation field. Most likely these features are due to very small dust grains or large molecules that are transiently heated to high temperature. From the PAH features that are present in the spectrum (especially at $3.3 \mu \mathrm{m}$ ) it is clear that a family of aromatic molecules is present. The plateau features may well be carried by an "extended PAH family" of aromatic molecules and clusters with attached aliphatic sides groups.

\subsection{Magnesium sulfide}

We find that the amount of $\mathrm{MgS}$ required to explain the strength of the " 30 " $\mu \mathrm{m}$ feature is consistent with the measured abundances of $\mathrm{Mg}$ and $\mathrm{S}$. Taking into account the envelope mass that we derived above $\left(0.44 M_{\odot}\right.$ for $\left.M_{\text {gas }} / M_{\text {dust }}=600\right)$ we conclude that only 25 per cent of the $\mathrm{Mg}$ - and S-atoms need to be condensed into $\mathrm{MgS}$ to explain the strength of the feature. Because HD 56126 is metal-poor this implies that in other sources that are generally more metal-rich the strength of the " 30 " $\mu \mathrm{m}$ feature is also consistent with the $\mathrm{MgS}$ identification.

\subsection{Titanium carbide}

We have put TiC in our model. The optical properties of nanocrystalline $\mathrm{TiC}$ are too poorly understood to accept or reject the TiC identification for the " 21 " $\mu \mathrm{m}$ feature. We do note clearly that the bulk optical properties of $\mathrm{TiC}$ are incompatible with 
the peak position, feature-over-continuum ratio and strength of the " $21 " \mu \mathrm{m}$ feature. The opacities of nano-TiC need to be $\sim 20$ times higher in the visible than measured in the bulk in order to explain the strength of the feature. It is interesting to note that a very similar resonance has been found in other nanocrystalline metal carbides, like VC, NbC (von Helden et al. 2001; van Heijnsbergen et al. 2002). Of course, these species will not contribute much to the " 21 " $\mu \mathrm{m}$ feature because of the much lower elemental abundance of $\mathrm{Nb}$ and $\mathrm{V}$. Better understanding the physics that gives rise to this resonance in nanocarbides may give further insight into the identification of the " 21 " $\mu \mathrm{m}$ feature. We conclude that the question of the identification of the " 21 " $\mu \mathrm{m}$ feature is still open and that nanocrystalline metal carbides remain very interesting candidates. Given the abundance of $\mathrm{Fe}$ and $\mathrm{Ni}$, cubic iron-, nickel carbides (haxonite) may be good first candidates for future studies.

\section{Summary and conclusions}

- We have presented a detailed study of the circumstellar envelope of the post-AGB star HD 56126. We present a large body of data from the literature, ISO spectroscopy and IR imaging. From a simple energy balance consideration we find a high effective temperature $7250 \pm 250 \mathrm{~K}$ for the central star consistent with the temperature derived from detailed abundance analyses.

- We have built a detailed dust radiative transfer model of the circumstellar envelope of HD 56126. To model the emission of the dust we use amorphous carbon (a-C), hydrogenated amorphous carbon (HAC), magnesium sulfide $(\mathrm{MgS})$ and titanium carbide (TiC). We present a detailed parametrisation of the optical properties of $\mathrm{HAC}$ as a function of $\mathrm{H} / \mathrm{C}$ content.

- The radiative transfer model explains the ISO spectroscopy from $2-200 \mu \mathrm{m}$ in great detail. The dust mass we derive is consistent with the dust mass as previously derived by other authors. We find that the mid-IR imaging and spectroscopy is best reproduced by a single dust shell from 1.2 to $2.6^{\prime \prime}$ radius around the central star. This compact shell originates from a short period of very high mass-loss during which the mass-loss rate exceeded $10^{-4} M_{\odot} / y r$.

_ "Classical" HAC grains in radiative equilibrium with the local radiation field do not reach a high enough temperature to explain the strength of the 3.3-3.4 and 6-9 $\mu \mathrm{m}$ hydrocarbon features relative to the $11-17 \mu \mathrm{m}$ hydrocarbon features. Hence we conclude that these features are carried by very small dust grains (or large molecules) which fluctuate in temperature upon absorption of a single UV/visible photon.

- The strength of the " 30 " $\mu \mathrm{m}$ feature is compatible with its identification with $\mathrm{MgS}$. This further strengthens the $\mathrm{MgS}$ identification of the " 30 " $\mu \mathrm{m}$ feature. The MgS temperature, derived from the observed profile allows an accurate determination of the MgS mass. We conclude that about 25 per cent of the $\mathrm{Mg}$ and $\mathrm{S}$ are locked up in $\mathrm{MgS}$ grains.

- We find that the observed strength of the " 21 " $\mu \mathrm{m}$ feature poses a problem for the $\mathrm{TiC}$ identification. The low abundance of $\mathrm{Ti}$ requires very high absorption crosssections in the UV and visible wavelength range to explain the strength of the feature. Laboratory studies of the UV absorption properties of TiC nano-crystals are therefore urgently needed. Other nano-crystalline metal carbides also exhibit similar resonances as found in nano-TiC and these metal carbides should also be investigated in the laboratory to clarify the nature of these resonances and the identification of the " 21 " $\mu \mathrm{m}$ feature.

Acknowledgements. We thank Margaret Meixner and Mike Jura for the helpful discussions and kindly supplying their mid-IR images. We thank Sun Kwok and Kevin Volk for kindly supplying the Gemini/OSCIR images we used in our analysis. We thank Hans van Winckel for providing his photometric data. We are very thankful to John Robertson and Uwe Kreibig for very useful directions, explanations and discussions on the properties of HAC and nanometals, respectively. We thank Tsuneharu Koide for supplying us with the optical constants of TiC. SH and LBFMW acknowledge financial support from an NWO Pionier grant (grant number 616-78-333). This research has made use of the SIMBAD database, operated at CDS, Strasbourg, France. This research has made use of NASA's Astrophysics Data System Bibliographic Services. IA $^{3}$ is a joint development of the SWS consortium. Contributing institutes are SRON, MPE, KUL and the ESA Astrophysics Division.

\section{References}

Allamandola, L. J., Tielens, A. G. G. M., \& Barker, J. R. 1989, ApJS, 71,733

Alvarez, M. M., Khoury, J. T., Schaaff, T. G., et al. 1997, J. Phys. Chem. B, 101, 3706

Alward, J. F., Fong, C. Y., El-Batanouny, M., \& Wooten, F. 1975, Phys. Rev. B, 12, 1105

Anders, E., \& Zinner, E. 1993, Meteoritics, 28, 490

Angus, J. C., Koidl, P., \& Domitz, S. 1986, Plasma Deposition of Thin Films (Boca Raton, FL: CRC Press), 89-127

Begemann, B., Dorschner, J., Henning, T., \& Mutschke, H. 1996, ApJ, 464, L195

Begemann, B., Dorschner, J., Henning, T., Mutschke, H., \& Thamm, E. 1994, ApJ, 423, L71

Bernatowicz, T. J., Cowsik, R., Gibbons, P. C., et al. 1996, ApJ, 472, 760

Bhattacharya, S. P., Wall, T. F., \& Gupta, R. P. 1996, Int. Comm. Heat Mass Transfer, 23, 809

Bogaert, E. 1994, Ph.D. Thesis, K.U. Leuven

Bohren, C. F., \& Huffman, D. R. 1983, Absorption and scattering of light by small particles (New York: Wiley)

Boothroyd, A. I., \& Sackmann, I.-J. 1988, ApJ, 328, 641

Bounouh, Y., Thèye, M. L., Dehbi-Alaoui, A., Matthews, A., \& Stoquert, J. P. 1995, Phys. Rev. B, 51, 9597

Bouwman, J. 2001, Ph.D. Thesis, University of Amsterdam

Bouwman, J., de Koter, A., van den Ancker, M. E., \& Waters, L. B. F. M. 2000, A\&A, 360, 213

Brewster, M. Q., \& Kunitomo, T. 1984, J. Heat Transfer, 106, 678

Busani, R., Folkers, M., \& Cheshnovsky, O. 1998, Phys. Rev. Lett., 81,3836

Buss, R. H., J., Cohen, M., Tielens, A. G. G. M., et al. 1990, ApJ, 365, L23

Černý, J. 1996, Fuel, 75, 1301

Clegg, P. E., Ade, P. A. R., Armand, C., et al. 1996, A\&A, 315, L38

Compagnini, G., Zammit, U., Madhusoodanan, K. N., \& Foti, G. 1995, Phys. Rev. B, 51, 11168 
Dayal, A., Hoffmann, W. F., Bieging, J. H., et al. 1998, ApJ, 492, 603 de Graauw, T., Haser, L. N., Beintema, D. A., et al. 1996, A\&A, 315, L49

de Jager, C., \& Nieuwenhuijzen, H. 1987, A\&A, 177, 217

Delin, A., Eriksson, O., Ahuja, R., et al. 1996, Phys. Rev. B, 54, 1673

Dischler, B. 1987, in E-MRS Symp. Proc., vol. XVII, Amorphous Hydrogen Carbon Films, ed. P. Koidl, \& P. Oelhafen (Les Ulis: Les Éditions de Physique), 189

Dischler, B., Bubenzer, A., \& Koidl, P. 1983, Appl. Phys. Lett., 42, 636

Dominik, C., Sedlmayr, E., \& Gail, H.-P. 1989, A\&A, 223, 227

Duley, W. W. 1984, ApJ, 287, 694

Espinosa-Magaña, F., Duarte-Moller, A., Martínez-Sánchez, R., \& Miki-Yoshida, M. 2002, J. Electr. Spectr. Related Phenom., 125, 119

Goebel, J. H. 1993, A\&A, 278, 226

Grishko, V. I., Tereszchuk, K., Duley, W. W., \& Bernath, P. 2001, ApJ, 558, L129

Guillois, O., Nenner, I., Papoular, R., \& Reynaud, C. 1994, A\&A, 285, 1003

Guillois, O., Nenner, I., Papoular, R., \& Reynaud, C. 1996, ApJ, 464, 810

Henning, T., \& Mutschke, H. 2001, Spectrochim. Acta A, 57, 815

Hill, H. G. M., Jones, A. P., \& D'Hendecourt, L. 1998, A\&A, 336, L41

Hony, S., Waters, L. B. F. M., \& Tielens, A. G. G. M. 2001, A\&A, 378, L41

Hony, S., Waters, L. B. F. M., \& Tielens, A. G. G. M. 2002, A\&A, 390,533

Hrivnak, B. J. 1995, ApJ, 438, 341

Hrivnak, B. J., Kwok, S., \& Volk, K. M. 1989, ApJ, 346, 265

Hrivnak, B. J., Volk, K., \& Kwok, S. 2000, ApJ, 535, 275

Ibarra, J. V., Munõz, E., \& Moliner, R. 1996, Organ. Geochem., 24, 725

Ihara, H., Kumashiro, Y., \& Itoh, A. 1975, Phys. Rev. B, 12, 5465

Jura, M. 1986, ApJ, 303, 327

Jura, M., Chen, C., \& Werner, M. W. 2000, ApJ, 544, L141

Justtanont, K., Barlow, M. J., Skinner, C. J., et al. 1996, A\&A, 309, 612

Käufl, H. U., Ageorges, N., Dietzsch, E., et al. 2000, The Messenger, 102,4

Kessler, M. F., Steinz, J. A., Anderegg, M. E., et al. 1996, A\&A, 315, L27

Klochkova, V. G. 1995, MNRAS, 272, 710

Knapp, G. R., Crosas, M., Young, K., \& Ivezić, C. 2000, ApJ, 534, 324

Knapp, G. R., Young, K., Lee, E., \& Jorissen, A. 1998, ApJS, 117, 209

Koide, T., Shidara, T., Fukutani, H., et al. 1990, Phys. Rev. B, 42, 4979

Koide, T., Shidara, T., Fukutani, H., et al. 1993, Jpn. J. Appl. Phys., 32,1130

Kreibig, U., \& Fragstein, C. v. 1969, Z. Phys., 224, 307

Kreibig, U., \& Vollmer, M. 1995, Optical properties of metal clusters, Springer Series in Material Science, No. 25 (Berlin: Springer-Verlag)

Kurucz, R. 1993, Kurucz CD-ROM No. 3. Cambridge, Mass., Smithsonian Astrophysical Observatory, 3

Kurucz, R. L. 1991, in NATO ASIC Proc. 341: Stellar atmospheres - beyond classical models, 441
Kwok, S., Hrivnak, B. J., \& Geballe, T. R. 1990, ApJ, 360, L23

Kwok, S., Volk, K., \& Bernath, P. 2001, ApJ, 554, L87

Kwok, S., Volk, K., \& Hrivnak, B. J. 1999, in Asymptotic Giant Branch stars, IAU Symp., 191, 297

Kwok, S., Volk, K., \& Hrivnak, B. J. 2002, ApJ, 573, 720

Kwok, S., Volk, K. M., \& Hrivnak, B. J. 1989, ApJ, 345, L51

Lye, R. G., \& Logothetis, E. M. 1966, Phys. Rev., 147, 622

Lynch, D. W., Olson, C. G., Peterman, D. J., \& Weaver, J. H. 1980, Phys. Rev. B, 22, 3991

Manickavasagam, S. 1993, Ph.D. Thesis, University of Kentucky

Mathis, J. S., Rumpl, W., \& Nordsieck, K. H. 1977, ApJ, 217, 425

Meixner, M., Skinner, C. J., Graham, J. R., et al. 1997, ApJ, 482, 897

Moutou, C., Leger, A., \& D’Hendecourt, L. 1996, A\&A, 310, 297

Nassau, J. J., Stephenson, C. B., \& McConnell, D. J. 1965, Hamburger Sternw. Warner \& Swasey Obs., 0

Olsen, E. H. 1993, A\&AS, 102, 89

Omont, A., Moseley, S. H., Cox, P., et al. 1995, ApJ, 454, 819

Oudmaijer, R. D., Waters, L. B. F. M., van Der Veen, W. E. C. J., \& Geballe, T. R. 1995, A\&A, 299, 69

Papoular, R. 2000, A\&A, 362, L9

Parthasarathy, M., Garcia Lario, P., \& Pottasch, S. R. 1992, A\&A, 264, 159

Pel, J. W. 1976, A\&AS, 24, 413

Pflüger, J., Fink, J., Weber, W., Bohnen, K. P., \& Crecelius, G. 1984, Phys. Rev. B, 30, 1155

Preibisch, T., Ossenkopf, V., Yorke, H. W., \& Henning, T. 1993, A\&A, 279, 577

Puente, G., d. 1., Iglesias, M. J., Fuente, E., \& Pis, J. J. 1998, J. Anal. Appl. Pyrolysis, 47, 33

Reimann, H., Linz, H., Wagner, R., et al. 2000, Proc. SPIE, 4008, 1132

Robertson, J. 1986, Adv. Phys., 35, 317

Robertson, J. 2002, Mat. Sci. Eng.: R, 37, 129

Savage, B. D., \& Mathis, J. S. 1979, ARA\&A, 17, 73

Schutte, W. A., Tielens, A. G. G. M., \& Allamandola, L. J. 1993, ApJ, 415, 397

Scott, A. D., Duley, W. W., \& Jahani, H. R. 1997, ApJ, 490, L175

Skinner, C. J., Meixner, M., Barlow, M. J., et al. 1995, Ap\&SS, 224, 383

Sourisseau, C., Coddens, G., \& Papoular, R. 1992, A\&A, 254, L1

Szczerba, R., Henning, T., Volk, K., Kwok, S., \& Cox, P. 1999, A\&A, 345, L39

Szczerba, R., Omont, A., Volk, K., Cox, P., \& Kwok, S. 1997, A\&A, 317,859

Tauc, J. 1973, Amorphous semiconductors (New York: Plenum)

Ueta, T., Meixner, M., \& Bobrowsky, M. 2000, ApJ, 528, 861

van Genderen, A. M., Greidanus, H., \& van Driel, W. 1986, A\&A, 155,72

van Heijnsbergen, D., Fielicke, A., Meijer, G., \& von Helden, G. 2002, Phys. Rev. Lett., 89, 013401

van Winckel, H., \& Reyniers, M. 2000, A\&A, 354, 135

Volk, K., Kwok, S., \& Hrivnak, B. J. 1999, ApJ, 516, L99

Volk, K., et al. 2002, IAU Symp., 209, in press

von Helden, G., Tielens, A. G. G. M., van Heijnsbergen, D., et al. 2000, Science, 288, 313

von Helden, G., van Heijnsbergen, D., Duncan, M. A., \& Meijer, G. 2001, Chem. Phys. Lett., 333, 350

Webster, A. 1995, MNRAS, 277, 1555

Yuasa, M., Unno, W., \& Magono, S. 1999, PASJ, 51, 197 